\title{
The Decarboxylation of $\alpha, \beta$-Unsaturated Acid Catalyzed by Prenylated FMN-dependent Ferulic Acid Decarboxylase and the Enzyme Inhibition
}

\author{
Cui-lan Lan and Shi-Lu Chen* \\ Key Laboratory of Cluster Science of Ministry of Education, School of \\ Chemistry and Chemical Engineering, Beijing Institute of Technology, Beijing \\ 100081, China \\ *E-mail: shlchen@bit.edu.cn
}

\section{Contents:}

Figure S1. Entropy-corrected PES for the reaction of Fdc1 with $\alpha$-methylcinnamic acid $\cdots \cdots \cdots \cdots \cdots$, S2

Figure S2. Entropy-corrected energetics for the reaction of Fdc1 with $\alpha$-hydroxycinnamic acid $\cdots \cdots$ S3

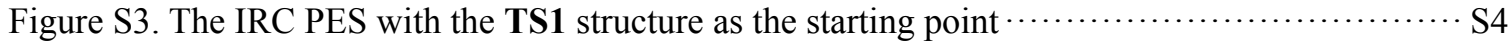

Figure S4. The IRC PES with the TS2 structure as the starting point $\cdots \cdots \cdots \cdots \cdots \cdots \cdots \cdots \cdots \cdots \cdots \cdots \cdots \cdots \cdots \cdots \cdots$

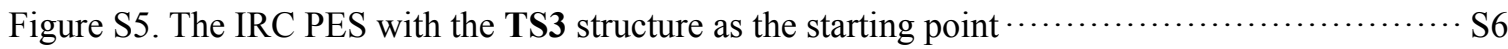

Figure S6. The IRC PES with the TS4 structure as the starting point $\cdots \cdots \cdots \cdots \cdots \cdots \cdots \cdots \cdots \cdots \cdots \cdots \cdots \cdots \cdots \cdots \cdots$

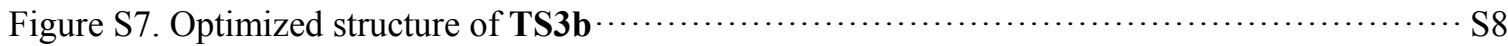

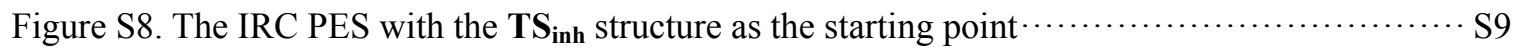

Figure S9. The IRC PES with the TS3c structure as the starting point $\cdots \cdots \cdots \cdots \cdots \cdots \cdots \cdots \cdots \cdots \cdots \cdots \cdots \cdots \cdots \cdots \cdots \cdots \cdots$

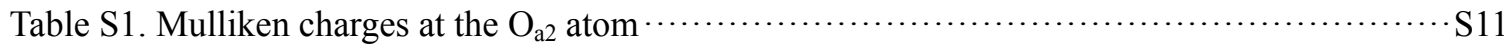

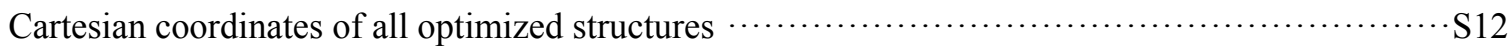




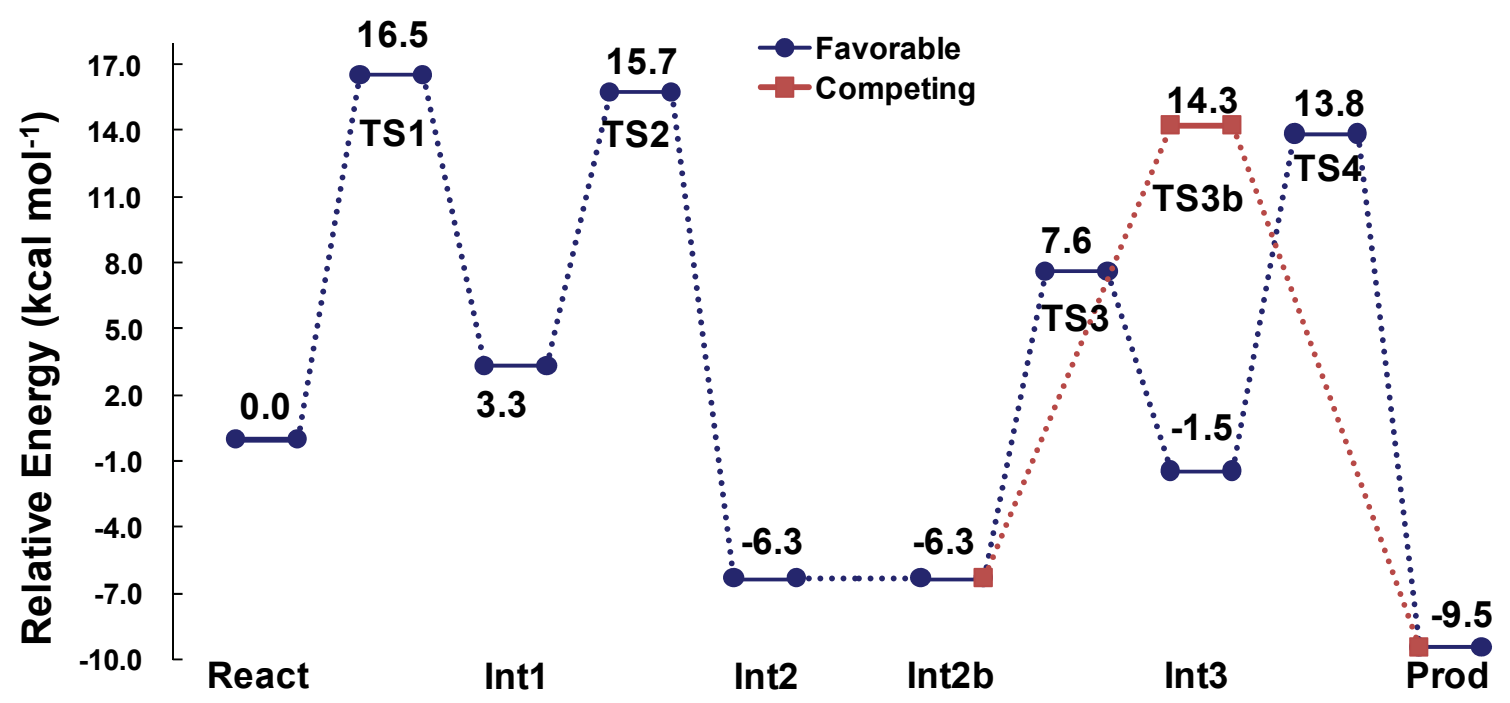

Figure S1. The entropy-corrected potential energy profile for the Fdc1-catalyzed decarboxylation of $\alpha$-methylcinnamic acid. 


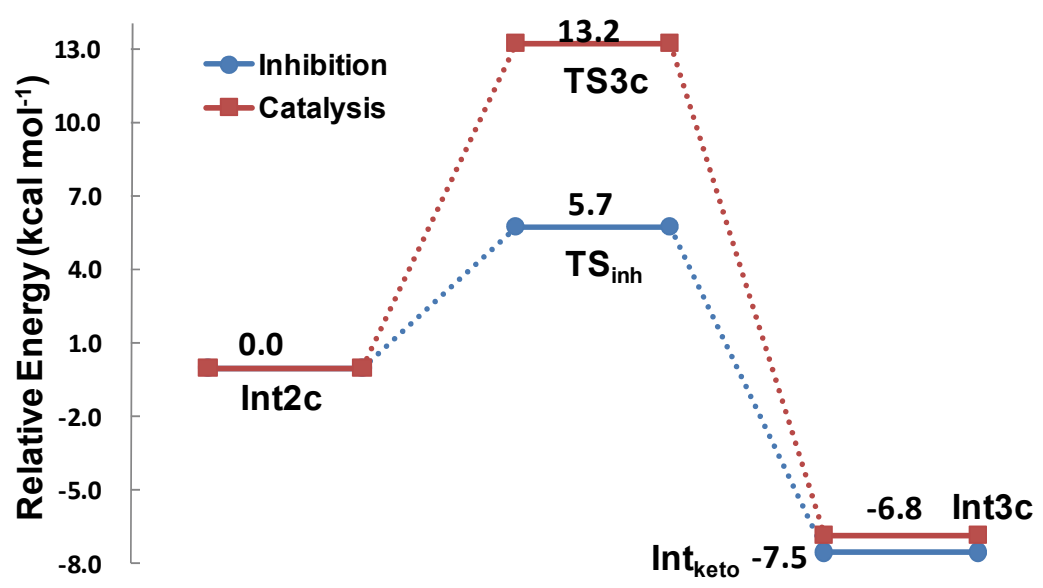

Figure S2. The entropy-corrected energetics for the Fdc1 reaction with $\alpha$-hydroxycinnamic acid. 


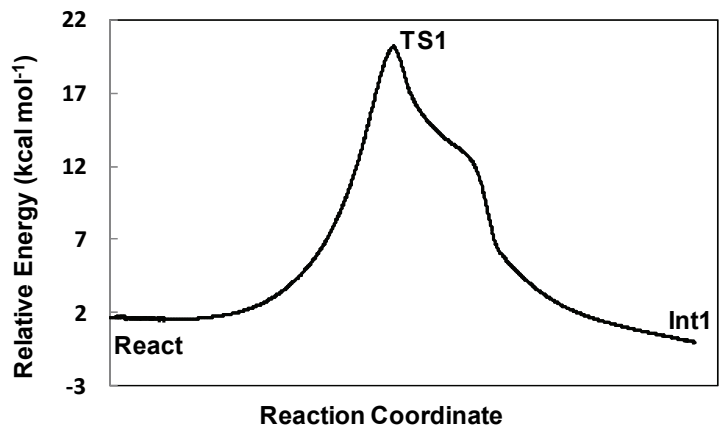

Figure S3. Schematic potential energy surface obtained by the IRC (intrinsic reaction coordinate) calculations with the TS1 structure as the starting point. Since the energies were computed at the same theory level as the geometry optimizations, they were not corrected for ZPE and solvation effects. However, dispersion effects were included in the energies, because the IRC calculations were performed using the B3LYP-D3 method. The IRC results given in Figures S4-S6, S8, and S9 follow the same pattern as Figure S3. 


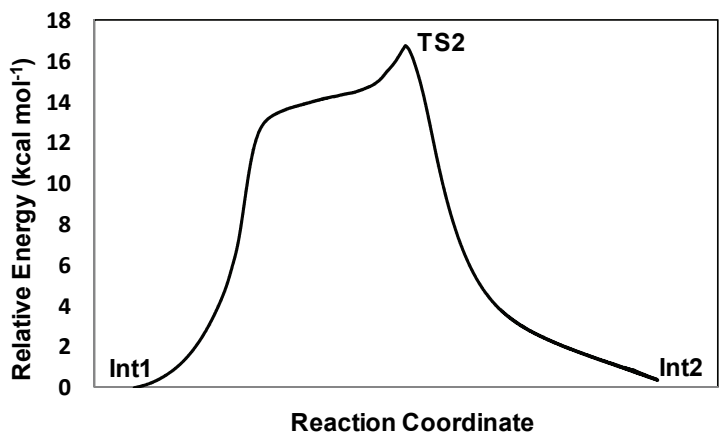

Figure S4. Schematic potential energy surface obtained by the IRC calculations with the TS2 structure as the starting point. 


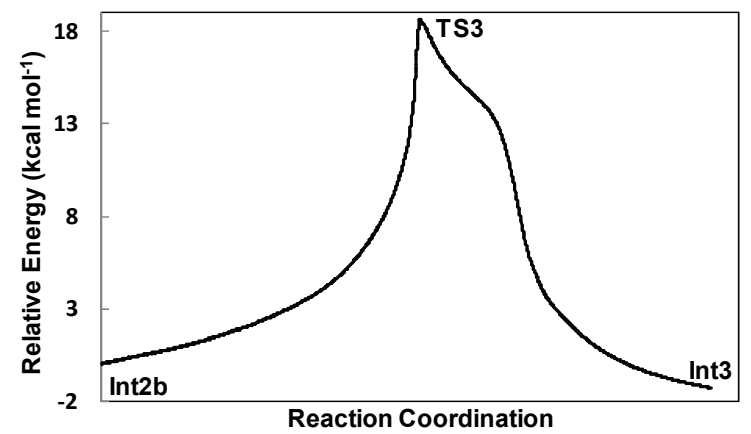

Figure S5. Schematic potential energy surface obtained by the IRC calculations with the TS3 structure as the starting point. 


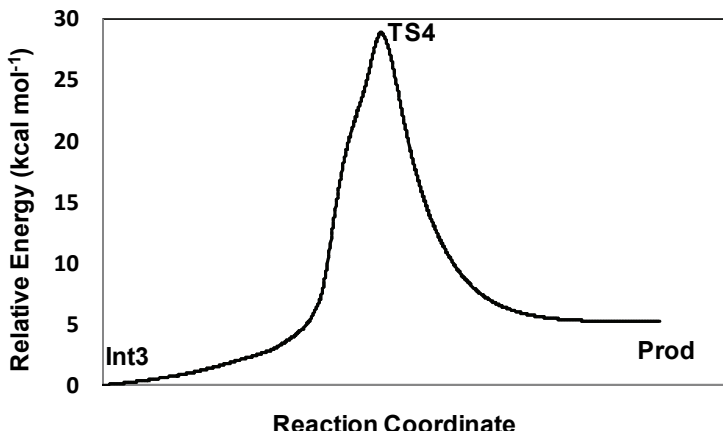

Figure S6. Schematic potential energy surface obtained by the IRC calculations with the TS4 structure as the starting point. 


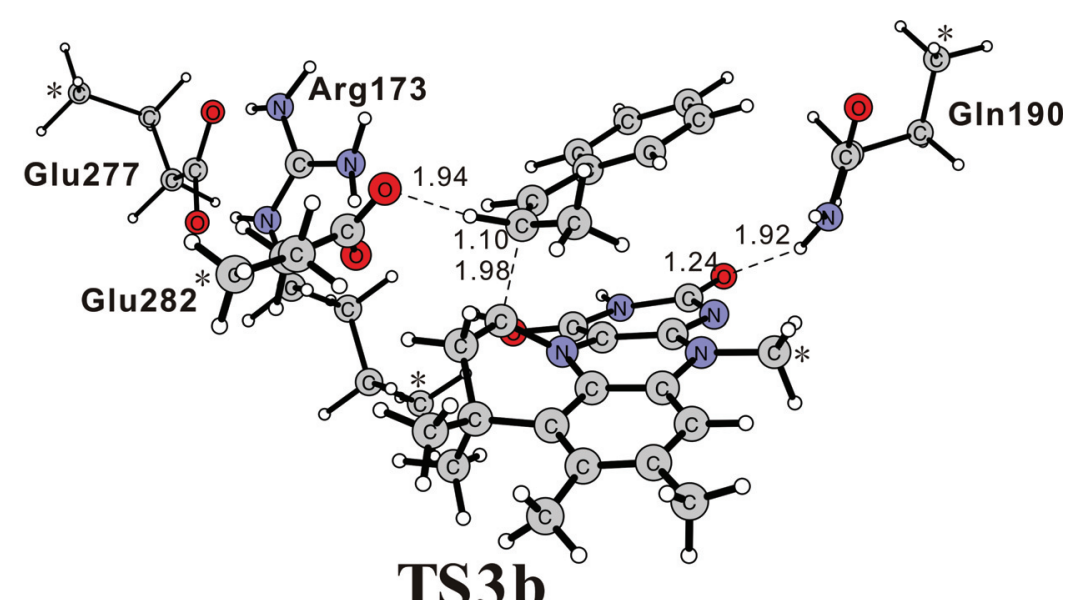

Figure S7. Optimized transition state (TS3b) for the decomposition of styrene derivative (Int2b) directly leading to the styrene product (Prod). TS3b has an imaginary frequency of $396 i \mathrm{~cm}^{-1}$. 


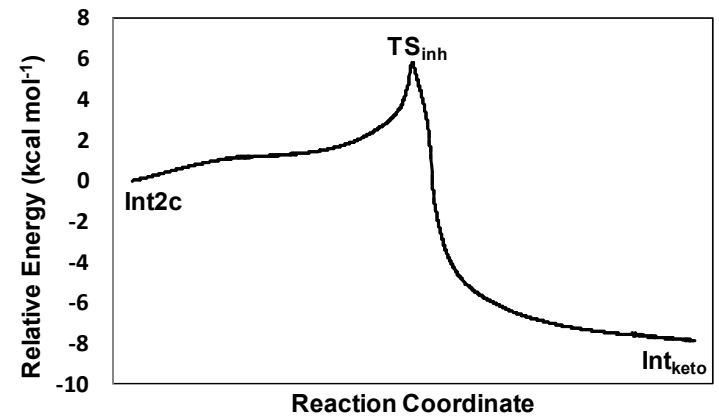

Figure S8. Schematic potential energy surface obtained by the IRC calculations with the $\mathbf{T S}_{\text {inh }}$ structure as the starting point. 


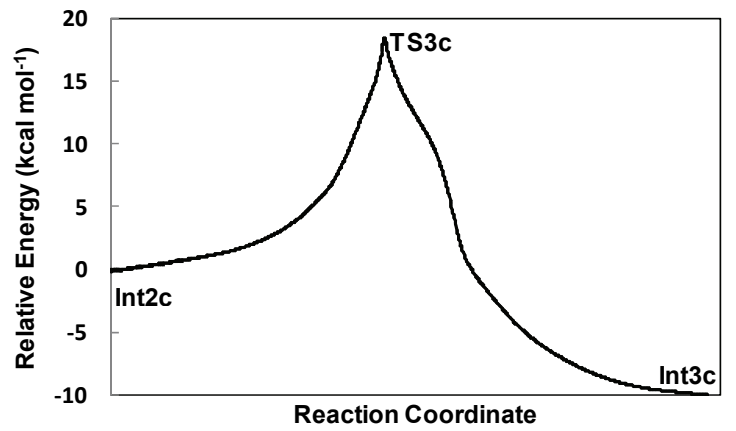

Figure S9. Schematic potential energy surface obtained by the IRC calculations with the TS3c structure as the starting point. 
Table S1. Mulliken charges at the $\mathrm{O}_{\mathrm{a} 2}$ atom in the Fdc1-catalyzed decarboxylation of $\alpha$-methylcinnamic acid

\begin{tabular}{lllllllllll}
\hline Complex & React & TS1 & Int1 & TS2 & Int2 & Int2b & TS3 & Int3 & TS4 & Prod \\
\hline Charge & -0.58 & -0.58 & -0.53 & -0.59 & -0.62 & -0.61 & -0.59 & -0.53 & -0.57 & -0.58 \\
\hline
\end{tabular}




\section{Cartesian coordinates of all optimized structures}

The abbreviations used here are consistent with those in the text.

\section{React}

$\begin{array}{lrrr}\mathrm{C} & 23.14199700 & 16.68498600 & 22.83199900 \\ \mathrm{C} & 21.64151200 & 16.96347700 & 22.94653900 \\ \mathrm{C} & 21.28190700 & 17.78562900 & 24.18874300 \\ \mathrm{C} & 19.78500100 & 18.10395400 & 24.24637800 \\ \mathrm{~N} & 19.48086600 & 18.99772100 & 25.35708600 \\ \mathrm{C} & 18.38681200 & 18.93910200 & 26.11601300 \\ \mathrm{~N} & 17.52610400 & 17.90858600 & 26.01966500 \\ \mathrm{~N} & 18.12512100 & 19.98215900 & 26.93401700 \\ \mathrm{C} & 26.03299900 & 8.54200500 & 31.72100100 \\ \mathrm{C} & 26.18386600 & 9.53532700 & 30.56511900 \\ \mathrm{C} & 24.84970200 & 9.85387300 & 29.85935000 \\ \mathrm{C} & 24.30598800 & 8.64231300 & 29.10395300 \\ \mathrm{O} & 23.65873700 & 7.75503100 & 29.66630000 \\ \mathrm{~N} & 24.65037100 & 8.59234100 & 27.79021700 \\ \mathrm{C} & 20.93700100 & 24.79701700 & 27.85200800 \\ \mathrm{C} & 22.01790900 & 23.73177600 & 28.06360700 \\ \mathrm{C} & 22.19551200 & 22.80443900 & 26.85721000 \\ \mathrm{C} & 21.03300000 & 21.82326700 & 26.59483100 \\ \mathrm{O} & 20.09010200 & 21.78883200 & 27.43748900 \\ \mathrm{O} & 21.13997000 & 21.11708400 & 25.55270500 \\ \mathrm{C} & 20.04317500 & 9.09493700 & 22.39811200 \\ \mathrm{C} & 19.25723000 & 9.37863300 & 21.28854700 \\ \mathrm{C} & 18.88875300 & 10.70967900 & 20.99052200 \\ \mathrm{C} & 22.16813400 & 10.86655300 & 24.80574300 \\ \mathrm{C} & 19.22552300 & 11.73727000 & 21.89698500 \\ \mathrm{C} & 24.00231300 & 11.48226800 & 26.07446300 \\ \mathrm{C} & 19.76193500 & 14.01757200 & 20.95249500 \\ \mathrm{C} & 18.74198000 & 13.19996700 & 21.77614500 \\ \mathrm{C} & 19.87856500 & 13.57343300 & 23.99713500 \\ \mathrm{C} & 25.00312900 & 11.24758000 & 26.77033300 \\ \mathrm{O} & 23.24058400 & 10.51255000 & 25.50048900 \\ \mathrm{~N} & 22.52778800 & 13.26379700 & 25.14465900 \\ \mathrm{O} & 22.29482300 & 14.47732300 & 25.06541600 \\ \mathrm{C} & 21.75663000 & 12.19022800 & 24.57382300 \\ \mathrm{C} & 17.32016100 & 13.38035200 & 21.19556500 \\ \mathrm{C} & 22.9791400 & 11.42441000 & 22.98171300 \\ \mathrm{C} & 10.96990900 & 19.67399700 \\ \mathrm{C} & 23.23398300\end{array}$




\begin{tabular}{|c|c|c|c|}
\hline $\mathrm{N}$ & 21.41047900 & 9.83192300 & 24.26324700 \\
\hline $\mathrm{C}$ & 21.71200300 & 8.44999200 & 24.62399200 \\
\hline $\mathrm{O}$ & 18.43561000 & 15.38472300 & 25.68443500 \\
\hline $\mathrm{C}$ & 17.49224300 & 14.73901400 & 26.26547900 \\
\hline $\mathrm{O}$ & 16.36518400 & 15.18702800 & 26.55857700 \\
\hline $\mathrm{C}$ & 17.80137700 & 13.28347400 & 26.63803200 \\
\hline $\mathrm{C}$ & 19.08692200 & 12.95594400 & 26.89051200 \\
\hline $\mathrm{C}$ & 19.67819200 & 11.67414500 & 27.29339800 \\
\hline $\mathrm{C}$ & 19.15760000 & 10.41119200 & 26.95121600 \\
\hline $\mathrm{C}$ & 19.78555800 & 9.23801100 & 27.37535700 \\
\hline $\mathrm{C}$ & 20.95285400 & 9.28774900 & 28.13984400 \\
\hline $\mathrm{C}$ & 21.49660500 & 10.53475900 & 28.45959100 \\
\hline $\mathrm{C}$ & 20.87545900 & 11.70369800 & 28.03689800 \\
\hline $\mathrm{H}$ & 21.10524200 & 16.00654900 & 22.98439500 \\
\hline $\mathrm{H}$ & 21.28942500 & 17.48668100 & 22.04488300 \\
\hline $\mathrm{H}$ & 21.56259800 & 17.22563500 & 25.08786200 \\
\hline $\mathrm{H}$ & 21.84901900 & 18.72542300 & 24.20126700 \\
\hline $\mathrm{H}$ & 19.22399000 & 17.17328700 & 24.34206200 \\
\hline $\mathrm{H}$ & 19.47563800 & 18.58184000 & 23.30278900 \\
\hline $\mathrm{H}$ & 20.09373700 & 19.85205100 & 25.45267800 \\
\hline $\mathrm{H}$ & 16.77347000 & 17.84675600 & 26.68944500 \\
\hline $\mathrm{H}$ & 17.88346700 & 16.95709200 & 25.76833500 \\
\hline $\mathrm{H}$ & 17.50473700 & 19.79765300 & 27.70732900 \\
\hline $\mathrm{H}$ & 18.87500600 & 20.70556900 & 27.09804100 \\
\hline $\mathrm{H}$ & 23.37492100 & 16.10516300 & 21.93067700 \\
\hline $\mathrm{H}$ & 26.61254100 & 10.47430500 & 30.93779800 \\
\hline $\mathrm{H}$ & 26.89153400 & 9.14659000 & 29.82200600 \\
\hline $\mathrm{H}$ & 24.10074600 & 10.12854900 & 30.61000100 \\
\hline $\mathrm{H}$ & 24.98261000 & 10.69394700 & 29.17066600 \\
\hline $\mathrm{H}$ & 24.89434300 & 9.45524000 & 27.29435700 \\
\hline $\mathrm{H}$ & 24.21255000 & 7.85811600 & 27.25296800 \\
\hline $\mathrm{H}$ & 25.56412900 & 7.61576400 & 31.37846000 \\
\hline $\mathrm{H}$ & 21.74853600 & 23.11907700 & 28.93089500 \\
\hline $\mathrm{H}$ & 22.97643600 & 24.21891500 & 28.29380700 \\
\hline $\mathrm{H}$ & 23.09637700 & 22.18815100 & 26.97492400 \\
\hline $\mathrm{H}$ & 22.35382600 & 23.38114800 & 25.93628800 \\
\hline $\mathrm{H}$ & 21.17869000 & 25.43976500 & 26.99559300 \\
\hline $\mathrm{H}$ & 20.33334400 & 8.06957300 & 22.58477300 \\
\hline $\mathrm{H}$ & 20.75817800 & 13.98465900 & 21.40344100 \\
\hline $\mathrm{H}$ & 19.45399100 & 15.06729900 & 20.90270100 \\
\hline $\mathrm{H}$ & 19.84128100 & 13.63314900 & 19.93100000 \\
\hline $\mathrm{H}$ & 20.23453300 & 14.32822100 & 24.67795300 \\
\hline $\mathrm{H}$ & 16.95147000 & 14.36983700 & 21.48226600 \\
\hline $\mathrm{H}$ & 16.62865400 & 12.63771000 & 21.60469600 \\
\hline
\end{tabular}




$\begin{array}{lrrr}\mathrm{H} & 17.28141200 & 13.33058400 & 20.10850100 \\ \mathrm{H} & 18.46617100 & 11.93115100 & 19.24701500 \\ \mathrm{H} & 17.09059700 & 10.95341900 & 19.76198700 \\ \mathrm{H} & 18.45579100 & 10.21144100 & 18.93784600 \\ \mathrm{H} & 22.28850200 & 8.47218300 & 25.54446900 \\ \mathrm{H} & 20.77773600 & 7.90924700 & 24.79283600 \\ \mathrm{H} & 19.79612800 & 13.77888600 & 26.83140700 \\ \mathrm{H} & 18.27304600 & 10.34819200 & 26.32761300 \\ \mathrm{H} & 19.35965600 & 8.27625300 & 27.09877700 \\ \mathrm{H} & 21.45041900 & 8.38422200 & 28.48040600 \\ \mathrm{H} & 22.41270900 & 10.59431000 & 29.03397700 \\ \mathrm{H} & 21.31377200 & 12.66743800 & 28.28252000 \\ \mathrm{H} & 25.39153900 & 8.95794900 & 32.50659000 \\ \mathrm{H} & 20.82555100 & 25.44141500 & 28.73237300 \\ \mathrm{H} & 19.97980500 & 24.30733200 & 27.65595500 \\ \mathrm{H} & 23.71169000 & 17.62068500 & 22.78482000 \\ \mathrm{H} & 23.48359300 & 16.11292400 & 23.69854400 \\ \mathrm{H} & 22.29710100 & 7.94709000 & 23.84436100 \\ \mathrm{H} & 27.00383600 & 8.30242600 & 32.16952500 \\ \mathrm{C} & 18.64922700 & 13.76674600 & 23.20746400 \\ \mathrm{H} & 17.82854800 & 13.27334700 & 23.75030700 \\ \mathrm{H} & 18.40352400 & 14.82957500 & 23.21058200 \\ \mathrm{C} & 18.83118600 & 8.22769700 & 20.40352700 \\ \mathrm{H} & 17.75526700 & 8.24687600 & 20.20067800 \\ \mathrm{H} & 19.34122800 & 8.24980900 & 19.43266500 \\ \mathrm{H} & 19.06574900 & 7.26943900 & 20.87382700 \\ \mathrm{~N} & 23.63884900 & 12.81102500 & 25.85009400 \\ \mathrm{H} & 24.20454100 & 13.52485100 & 26.29058100 \\ \mathrm{C} & 16.60982700 & 12.37656500 & 26.78646300 \\ \mathrm{H} & 16.34310700 & 11.90149400 & 25.83074000 \\ \mathrm{H} & 16.77806600 & 11.57826700 & 27.51528900 \\ \mathrm{H} & 15.75687100 & 12.98801600 & 27.08947100\end{array}$

TS1

$\begin{array}{lrrr}\mathrm{C} & 23.14195800 & 16.68500400 & 22.83202600 \\ \mathrm{C} & 21.66828100 & 17.05659300 & 23.00796300 \\ \mathrm{C} & 21.36871300 & 17.72800800 & 24.35133700 \\ \mathrm{C} & 19.89769400 & 18.14482600 & 24.45567600 \\ \mathrm{~N} & 19.66233400 & 18.94599500 & 25.65108700 \\ \mathrm{C} & 18.68437100 & 18.78172600 & 26.53296200 \\ \mathrm{~N} & 17.86389400 & 17.71154000 & 26.50164800 \\ \mathrm{~N} & 18.47340800 & 19.76294800 & 27.44144300 \\ \mathrm{C} & 26.03297800 & 8.54202000 & 31.72099200\end{array}$




\begin{tabular}{|c|c|c|c|}
\hline $\mathrm{C}$ & 25.65847200 & 9.73770700 & 30.83969800 \\
\hline $\mathrm{C}$ & 24.13733800 & 9.90659600 & 30.66644500 \\
\hline $\mathrm{C}$ & 23.49031300 & 8.69800000 & 29.99311900 \\
\hline $\mathrm{O}$ & 22.79528700 & 7.89141700 & 30.61553100 \\
\hline $\mathrm{N}$ & 23.78388100 & 8.56724200 & 28.67175900 \\
\hline $\mathrm{C}$ & 20.93702400 & 24.79708100 & 27.85201200 \\
\hline $\mathrm{C}$ & 22.10353000 & 23.84796700 & 28.14455800 \\
\hline $\mathrm{C}$ & 22.33834500 & 22.83062300 & 27.02463400 \\
\hline $\mathrm{C}$ & 21.22836100 & 21.77330300 & 26.85357200 \\
\hline $\mathrm{O}$ & 20.34582300 & 21.69640000 & 27.75695700 \\
\hline $\mathrm{O}$ & 21.31430200 & 21.05216400 & 25.81997000 \\
\hline $\mathrm{C}$ & 20.80347200 & 9.15894800 & 21.98927200 \\
\hline $\mathrm{C}$ & 20.35567000 & 9.48883300 & 20.71125000 \\
\hline $\mathrm{C}$ & 20.09022300 & 10.83391200 & 20.36679000 \\
\hline $\mathrm{C}$ & 22.13110300 & 10.84585800 & 24.97314000 \\
\hline $\mathrm{C}$ & 20.19080500 & 11.83481600 & 21.35910800 \\
\hline $\mathrm{C}$ & 23.55042800 & 11.43198800 & 26.70331300 \\
\hline $\mathrm{C}$ & 21.17236900 & 14.09467700 & 20.87218400 \\
\hline $\mathrm{C}$ & 19.86284800 & 13.33670600 & 21.18919600 \\
\hline $\mathrm{C}$ & 20.02558300 & 13.50273900 & 23.75862300 \\
\hline $\mathrm{O}$ & 24.32230700 & 11.17877100 & 27.64088800 \\
\hline $\mathrm{N}$ & 22.93527700 & 10.47021300 & 25.95671100 \\
\hline $\mathrm{C}$ & 22.51207400 & 13.24181300 & 25.33480500 \\
\hline $\mathrm{O}$ & 22.44239000 & 14.45373100 & 25.10382200 \\
\hline $\mathrm{C}$ & 21.81336300 & 12.18090100 & 24.64746500 \\
\hline $\mathrm{N}$ & 20.90215000 & 12.45870300 & 23.61716600 \\
\hline $\mathrm{C}$ & 18.80248500 & 13.68403500 & 20.12028600 \\
\hline $\mathrm{C}$ & 20.68797800 & 11.47040200 & 22.62785100 \\
\hline $\mathrm{C}$ & 19.77550400 & 11.14697300 & 18.91646200 \\
\hline $\mathrm{C}$ & 21.01374500 & 10.14190000 & 22.95723800 \\
\hline $\mathrm{N}$ & 21.52371500 & 9.83920400 & 24.22808400 \\
\hline $\mathrm{C}$ & 21.71204000 & 8.44989400 & 24.62397000 \\
\hline $\mathrm{O}$ & 18.82671200 & 15.30905100 & 25.70721400 \\
\hline $\mathrm{C}$ & 18.08059600 & 14.40177200 & 25.23359500 \\
\hline $\mathrm{O}$ & 16.93118000 & 14.47349500 & 24.78211100 \\
\hline $\mathrm{C}$ & 18.74554400 & 12.95176000 & 25.18896800 \\
\hline $\mathrm{C}$ & 19.57478800 & 12.69892500 & 26.29309000 \\
\hline $\mathrm{C}$ & 19.83360200 & 11.49972200 & 27.06369600 \\
\hline $\mathrm{C}$ & 19.43069800 & 10.18487100 & 26.72545100 \\
\hline $\mathrm{C}$ & 19.74176000 & 9.10684400 & 27.55049100 \\
\hline $\mathrm{C}$ & 20.46120400 & 9.28825900 & 28.73304100 \\
\hline $\mathrm{C}$ & 20.87283300 & 10.57782100 & 29.08297000 \\
\hline $\mathrm{C}$ & 20.57163300 & 11.65693000 & 28.26412800 \\
\hline $\mathrm{H}$ & 21.05895200 & 16.14980900 & 22.92737500 \\
\hline
\end{tabular}




\begin{tabular}{|c|c|c|c|}
\hline $\mathrm{H}$ & 21.35117800 & 17.71884300 & 22.18812900 \\
\hline $\mathrm{H}$ & 21.60387600 & 17.03218600 & 25.16409400 \\
\hline $\mathrm{H}$ & 21.99984200 & 18.61668100 & 24.48118600 \\
\hline $\mathrm{H}$ & 19.26503200 & 17.25600700 & 24.45752800 \\
\hline $\mathrm{H}$ & 19.62558400 & 18.74889700 & 23.57678800 \\
\hline $\mathrm{H}$ & 20.27809400 & 19.80072500 & 25.75787000 \\
\hline $\mathrm{H}$ & 17.15121300 & 17.66535800 & 27.21225000 \\
\hline $\mathrm{H}$ & 18.17658200 & 16.79274400 & 26.11230800 \\
\hline $\mathrm{H}$ & 18.01760800 & 19.48560200 & 28.29755300 \\
\hline $\mathrm{H}$ & 19.18791600 & 20.53538400 & 27.53159200 \\
\hline $\mathrm{H}$ & 23.32030900 & 16.21208100 & 21.85880400 \\
\hline $\mathrm{H}$ & 26.06590100 & 10.65965300 & 31.27366400 \\
\hline $\mathrm{H}$ & 26.11429300 & 9.63387000 & 29.84860600 \\
\hline $\mathrm{H}$ & 23.65299600 & 10.02204100 & 31.64098900 \\
\hline $\mathrm{H}$ & 23.94525800 & 10.79672000 & 30.05812800 \\
\hline $\mathrm{H}$ & 24.07048600 & 9.39784700 & 28.14375100 \\
\hline $\mathrm{H}$ & 23.24865900 & 7.86945000 & 28.17610700 \\
\hline $\mathrm{H}$ & 25.64571000 & 7.60773800 & 31.30226600 \\
\hline $\mathrm{H}$ & 21.89301000 & 23.29894500 & 29.06877400 \\
\hline $\mathrm{H}$ & 23.02208500 & 24.42879600 & 28.31081000 \\
\hline $\mathrm{H}$ & 23.26939900 & 22.27504800 & 27.19944200 \\
\hline $\mathrm{H}$ & 22.47029900 & 23.33026200 & 26.05646800 \\
\hline $\mathrm{H}$ & 21.11509700 & 25.37019100 & 26.93299700 \\
\hline $\mathrm{H}$ & 21.02250400 & 8.12294500 & 22.21514500 \\
\hline $\mathrm{H}$ & 21.90123600 & 13.99496000 & 21.68019200 \\
\hline $\mathrm{H}$ & 20.97074200 & 15.16179600 & 20.73459800 \\
\hline $\mathrm{H}$ & 21.62427800 & 13.70924000 & 19.95193600 \\
\hline $\mathrm{H}$ & 20.43194700 & 14.33206900 & 24.32146700 \\
\hline $\mathrm{H}$ & 18.45016300 & 14.70439900 & 20.30162800 \\
\hline $\mathrm{H}$ & 17.93509500 & 13.02037000 & 20.18395800 \\
\hline $\mathrm{H}$ & 19.18868200 & 13.65995600 & 19.10189300 \\
\hline $\mathrm{H}$ & 20.28632200 & 12.05262900 & 18.58683600 \\
\hline $\mathrm{H}$ & 18.70545200 & 11.28471000 & 18.72802600 \\
\hline $\mathrm{H}$ & 20.11463200 & 10.34333600 & 18.26185900 \\
\hline $\mathrm{H}$ & 21.94955100 & 8.43652700 & 25.68430200 \\
\hline $\mathrm{H}$ & 20.78693100 & 7.89543400 & 24.44524400 \\
\hline $\mathrm{H}$ & 20.02066300 & 13.60329200 & 26.70122700 \\
\hline $\mathrm{H}$ & 18.89376700 & 9.99932700 & 25.80808200 \\
\hline $\mathrm{H}$ & 19.42240000 & 8.10903800 & 27.25997600 \\
\hline $\mathrm{H}$ & 20.73065400 & 8.44907500 & 29.36671700 \\
\hline $\mathrm{H}$ & 21.44071100 & 10.73209100 & 29.99445800 \\
\hline $\mathrm{H}$ & 20.90788200 & 12.65247900 & 28.53948600 \\
\hline $\mathrm{H}$ & 25.60724300 & 8.64740800 & 32.72551200 \\
\hline $\mathrm{H}$ & 20.78257700 & 25.51366300 & 28.66771400 \\
\hline
\end{tabular}




$\begin{array}{lrrr}\mathrm{H} & 20.02019200 & 24.21627500 & 27.72300800 \\ \mathrm{H} & 23.77893500 & 17.57573200 & 22.89183700 \\ \mathrm{H} & 23.44972500 & 15.98479900 & 23.61221000 \\ \mathrm{H} & 22.53170300 & 7.97578200 & 24.06919800 \\ \mathrm{H} & 27.11977100 & 8.44579400 & 31.82189600 \\ \mathrm{C} & 19.23710700 & 13.84543800 & 22.51776900 \\ \mathrm{H} & 18.21976900 & 13.45509600 & 22.59447800 \\ \mathrm{H} & 19.13092800 & 14.93261800 & 22.47459900 \\ \mathrm{C} & 20.19041000 & 8.36502400 & 19.70942500 \\ \mathrm{H} & 19.20564500 & 8.38394600 & 19.23011900 \\ \mathrm{H} & 20.93861500 & 8.41303000 & 18.90840000 \\ \mathrm{H} & 20.30101400 & 7.39337900 & 20.19832900 \\ \mathrm{~N} & 23.31817300 & 12.76710000 & 26.36899800 \\ \mathrm{H} & 23.81677100 & 13.46762700 & 26.90193700 \\ \mathrm{C} & 17.86075600 & 11.87118300 & 24.59959700 \\ \mathrm{H} & 18.44168700 & 11.09951700 & 24.08584200 \\ \mathrm{H} & 17.26951800 & 11.38872700 & 25.38492700 \\ \mathrm{H} & 17.15005300 & 12.31633600 & 23.90467300\end{array}$

\section{Int1}

C

C

C

C

$\mathrm{N}$

C

$\mathrm{N}$

$\mathrm{N}$

C

C

C

C

$\mathrm{O}$

$\mathrm{N}$

C

C

C

C

$\mathrm{O}$

$\mathrm{O}$

C

C

C
23.14199600

22.01241600

22.22629800

21.05091700

21.13549100

20.55980700

19.95663200

20.54793500

26.03298800

25.97005500

24.55164100

23.56806900

22.73240300

23.73906600

20.93700200

22.18120300

23.17416700

22.54036900

21.85247400

22.74811700

20.56180300

19.99885100

19.82510700
16.68498400

17.62267400

18.19171600

19.06998700

19.50526100

18.86573800

17.68363600

19.47273600

8.54201100

9.64187600

10.20985200

9.14479800

8.63216100

8.76407400

24.79702800

24.64249200

23.62265900

22.22471100

21.82624800

21.60801800

9.15176800

9.46597800

10.81911700
22.83200000

23.26078100

24.66647100

25.11241700

26.49859200

27.51857500

27.34478200

28.72588300

31.72098400

30.65558000

30.45758200

29.98109800

30.72418700

28.68228300

27.85201200

28.73323000

28.16180900

28.02075900

29.00603400

26.93950900

22.10069300

20.86476300

20.49289300 


\begin{tabular}{|c|c|c|c|}
\hline $\mathrm{C}$ & 22.37994400 & 10.75461700 & 24.86201800 \\
\hline $\mathrm{C}$ & 20.16953200 & 11.84865300 & 21.39475600 \\
\hline $\mathrm{C}$ & 24.20984300 & 11.22651400 & 26.20310700 \\
\hline $\mathrm{C}$ & 21.16568600 & 14.08554600 & 20.74234400 \\
\hline $\mathrm{C}$ & 19.88998900 & 13.36228300 & 21.23123800 \\
\hline $\mathrm{C}$ & 20.54121900 & 13.70281300 & 23.69010000 \\
\hline $\mathrm{O}$ & 24.96570100 & 10.97220700 & 27.13630800 \\
\hline $\mathrm{N}$ & 23.25498300 & 10.34891500 & 25.74373500 \\
\hline $\mathrm{C}$ & 23.57393300 & 12.82531800 & 24.43692300 \\
\hline $\mathrm{O}$ & 23.90036900 & 13.64490600 & 23.60365400 \\
\hline $\mathrm{C}$ & 22.16425000 & 12.21149500 & 24.57012600 \\
\hline $\mathrm{N}$ & 21.33655400 & 12.49091200 & 23.42396000 \\
\hline $\mathrm{C}$ & 18.70998200 & 13.73281300 & 20.30644800 \\
\hline $\mathrm{C}$ & 20.83277600 & 11.49968000 & 22.60129900 \\
\hline $\mathrm{C}$ & 19.33868300 & 11.10258200 & 19.08368000 \\
\hline $\mathrm{C}$ & 20.99130200 & 10.15310000 & 22.96661700 \\
\hline $\mathrm{N}$ & 21.61795100 & 9.84296500 & 24.20826500 \\
\hline $\mathrm{C}$ & 21.71201400 & 8.44997700 & 24.62400300 \\
\hline $\mathrm{O}$ & 20.43006600 & 15.88048800 & 25.41707700 \\
\hline $\mathrm{C}$ & 19.73662000 & 14.89363700 & 25.83269800 \\
\hline $\mathrm{O}$ & 18.86373000 & 14.89760300 & 26.71539500 \\
\hline $\mathrm{C}$ & 20.10953300 & 13.52345700 & 25.16697700 \\
\hline $\mathrm{C}$ & 21.47464300 & 13.08089700 & 25.77339000 \\
\hline $\mathrm{C}$ & 21.47940700 & 12.52816000 & 27.17455000 \\
\hline $\mathrm{C}$ & 20.94285300 & 11.27855100 & 27.52606300 \\
\hline $\mathrm{C}$ & 20.96623600 & 10.83483300 & 28.84521100 \\
\hline $\mathrm{C}$ & 21.51206600 & 11.63736800 & 29.84885300 \\
\hline $\mathrm{C}$ & 22.03530900 & 12.88695200 & 29.51941700 \\
\hline $\mathrm{C}$ & 22.02253400 & 13.32260400 & 28.19429400 \\
\hline $\mathrm{H}$ & 21.06619100 & 17.06896500 & 23.26529900 \\
\hline $\mathrm{H}$ & 21.90616000 & 18.44374800 & 22.53592300 \\
\hline $\mathrm{H}$ & 22.32165500 & 17.35725900 & 25.36693200 \\
\hline $\mathrm{H}$ & 23.15453700 & 18.77679000 & 24.71412700 \\
\hline $\mathrm{H}$ & 20.11560300 & 18.52109700 & 24.96335400 \\
\hline $\mathrm{H}$ & 21.00533900 & 19.97369300 & 24.49236700 \\
\hline $\mathrm{H}$ & 21.77117400 & 20.31924100 & 26.71710500 \\
\hline $\mathrm{H}$ & 19.40794600 & 17.27519000 & 28.08391300 \\
\hline $\mathrm{H}$ & 20.13355400 & 17.05137100 & 26.52672600 \\
\hline $\mathrm{H}$ & 20.45089700 & 18.87544700 & 29.53178700 \\
\hline $\mathrm{H}$ & 21.06026600 & 20.38677300 & 28.84649400 \\
\hline $\mathrm{H}$ & 22.96557000 & 16.25772600 & 21.83929200 \\
\hline $\mathrm{H}$ & 26.64089600 & 10.46471200 & 30.93327500 \\
\hline $\mathrm{H}$ & 26.33350100 & 9.25599800 & 29.69598800 \\
\hline $\mathrm{H}$ & 24.16319300 & 10.59854000 & 31.40316300 \\
\hline
\end{tabular}




\begin{tabular}{|c|c|c|c|}
\hline $\mathrm{H}$ & 24.57795200 & 11.02632800 & 29.72851300 \\
\hline $\mathrm{H}$ & 24.20844900 & 9.39431300 & 28.03403800 \\
\hline $\mathrm{H}$ & 23.01390300 & 8.17270100 & 28.30440900 \\
\hline $\mathrm{H}$ & 25.37417500 & 7.70644800 & 31.46472400 \\
\hline $\mathrm{H}$ & 21.87561100 & 24.29643500 & 29.72622600 \\
\hline $\mathrm{H}$ & 22.67554900 & 25.61625100 & 28.85899000 \\
\hline $\mathrm{H}$ & 24.04083400 & 23.53605100 & 28.83119100 \\
\hline $\mathrm{H}$ & 23.54551100 & 23.93738800 & 27.18057600 \\
\hline $\mathrm{H}$ & 21.20723800 & 25.09868400 & 26.83261100 \\
\hline $\mathrm{H}$ & 20.68399100 & 8.10862700 & 22.36442700 \\
\hline $\mathrm{H}$ & 22.01878100 & 13.89149700 & 21.39677600 \\
\hline $\mathrm{H}$ & 20.99874100 & 15.16837900 & 20.71395600 \\
\hline $\mathrm{H}$ & 21.42540700 & 13.75505300 & 19.73046500 \\
\hline $\mathrm{H}$ & 21.21523400 & 14.56041600 & 23.65453600 \\
\hline $\mathrm{H}$ & 18.44081600 & 14.77793000 & 20.48966000 \\
\hline $\mathrm{H}$ & 17.82677300 & 13.12126700 & 20.51606900 \\
\hline $\mathrm{H}$ & 18.94531000 & 13.65073200 & 19.24566300 \\
\hline $\mathrm{H}$ & 19.85404500 & 11.96114200 & 18.65227500 \\
\hline $\mathrm{H}$ & 18.26265400 & 11.30197000 & 19.03180300 \\
\hline $\mathrm{H}$ & 19.53948200 & 10.25520400 & 18.42656300 \\
\hline $\mathrm{H}$ & 22.06544500 & 8.42280400 & 25.65133900 \\
\hline $\mathrm{H}$ & 20.72433300 & 7.98929800 & 24.55487900 \\
\hline $\mathrm{H}$ & 22.06109900 & 14.00395200 & 25.79692800 \\
\hline $\mathrm{H}$ & 20.51501000 & 10.64074000 & 26.76102100 \\
\hline $\mathrm{H}$ & 20.58722000 & 9.85000300 & 29.09889000 \\
\hline $\mathrm{H}$ & 21.55155600 & 11.26614000 & 30.86746200 \\
\hline $\mathrm{H}$ & 22.46380700 & 13.52114700 & 30.29026700 \\
\hline $\mathrm{H}$ & 22.42665700 & 14.29912500 & 27.94213600 \\
\hline $\mathrm{H}$ & 25.70684100 & 8.92335900 & 32.69535500 \\
\hline $\mathrm{H}$ & 20.24728100 & 25.54898200 & 28.25372700 \\
\hline $\mathrm{H}$ & 20.40154200 & 23.84505800 & 27.79125900 \\
\hline $\mathrm{H}$ & 24.10404600 & 17.21206600 & 22.80094600 \\
\hline $\mathrm{H}$ & 23.24494000 & 15.85270500 & 23.52934800 \\
\hline $\mathrm{H}$ & 22.41709400 & 7.89213000 & 23.99746700 \\
\hline $\mathrm{H}$ & 27.05123900 & 8.15334600 & 31.83272600 \\
\hline $\mathrm{C}$ & 19.46416100 & 13.87685500 & 22.63793000 \\
\hline $\mathrm{H}$ & 18.55013000 & 13.34479600 & 22.91766600 \\
\hline $\mathrm{H}$ & 19.21488700 & 14.94100300 & 22.58808200 \\
\hline $\mathrm{C}$ & 19.59646100 & 8.32583800 & 19.95281200 \\
\hline $\mathrm{H}$ & 18.56489300 & 8.42424500 & 19.59617100 \\
\hline $\mathrm{H}$ & 20.23628800 & 8.25525700 & 19.06352700 \\
\hline $\mathrm{H}$ & 19.67033100 & 7.36908000 & 20.47803900 \\
\hline $\mathrm{N}$ & 24.40456100 & 12.40835000 & 25.46252300 \\
\hline $\mathrm{H}$ & 25.27981600 & 12.89737200 & 25.60197300 \\
\hline
\end{tabular}




$\begin{array}{llll}\mathrm{C} & 18.98720700 & 12.50331600 & 25.35860600 \\ \mathrm{H} & 19.22411700 & 11.53107100 & 24.91119700 \\ \mathrm{H} & 18.78927200 & 12.37839000 & 26.42213100 \\ \mathrm{H} & 18.06048400 & 12.87190400 & 24.91313000\end{array}$

\section{TS2}

$\mathrm{C}$

23.14190700

16.68444900

22.83211100

C

21.84502400

17.29771100

23.36456300

C

21.94888800

17.70363500

24.83756500

C

$\mathrm{N}$

20.70294600

18.46064400

25.31362200

20.84805600

19.00246600

26.66075900

20.60036900

18.35405600

27.78446300

20.22928300

17.04100900

27.80282300

20.71408400

19.01262700

28.95320700

26.03299600

8.54201000

31.72099700

25.69482200

9.73529900

30.81964000

24.19672100

10.08917400

30.81233800

23.29978300

8.97026200

30.28421700

22.44951800

8.41864600

30.98715000

23.53091200

8.62866900

28.98756500

20.93699900

24.79702900

27.85201300

22.21548800

24.35547800

28.57278500

22.95983200

23.24183100

27.82635100

22.19763900

21.90418600

27.80833100

21.67398700

21.53314100

28.89858200

22.17911800

21.27554600

26.71071300

20.75209000

9.26667500

22.05436200

20.23051500

9.65295000

20.81751100

19.95614100

11.01162300

20.55240300

22.26342700

10.79125800

25.05120800

20.15608600

11.97251300

21.57149600

23.78708100

11.25742800

26.72931800

21.03258000

14.31634900

21.21080400

19.77972400

13.47101000

21.52732500

20.14914000

13.56314200

24.10054300

24.52248700

10.94898500

27.68336600

23.05496400

10.34762900

26.02242500

22.91858000

13.12798500

25.32494100

22.95733200

14.34409600

25.07694100

22.06072500

12.14791700

24.72039900

21.11211400

12.51223100

23.74833600

18.65310400

13.85535500

20.54290000

20.75780600

11.56182000

22.78107900 


\begin{tabular}{|c|c|c|c|}
\hline $\mathrm{C}$ & 19.53250300 & 11.38895500 & 19.14458700 \\
\hline $\mathrm{C}$ & 21.04199300 & 10.20702300 & 23.04043700 \\
\hline $\mathrm{N}$ & 21.58005600 & 9.83998900 & 24.29204200 \\
\hline $\mathrm{C}$ & 21.71851100 & 8.42862500 & 24.61362900 \\
\hline $\mathrm{O}$ & 18.96555600 & 15.68401300 & 25.60968400 \\
\hline $\mathrm{C}$ & 18.32023300 & 14.64895400 & 25.72120100 \\
\hline $\mathrm{O}$ & 17.19840100 & 14.24918100 & 25.91405200 \\
\hline $\mathrm{C}$ & 19.46260300 & 13.11135700 & 25.42998100 \\
\hline $\mathrm{C}$ & 20.33596400 & 13.26761400 & 26.52094200 \\
\hline $\mathrm{C}$ & 20.50438500 & 12.54296100 & 27.76397500 \\
\hline $\mathrm{C}$ & 20.26919200 & 11.15934100 & 27.93497400 \\
\hline $\mathrm{C}$ & 20.54066600 & 10.53929500 & 29.14948400 \\
\hline $\mathrm{C}$ & 21.03193300 & 11.27556500 & 30.23097200 \\
\hline $\mathrm{C}$ & 21.28167100 & 12.64214300 & 30.07914200 \\
\hline $\mathrm{C}$ & 21.03925700 & 13.26246900 & 28.85922800 \\
\hline $\mathrm{H}$ & 21.02025400 & 16.58244700 & 23.25150300 \\
\hline $\mathrm{H}$ & 21.57462700 & 18.17866800 & 22.76322700 \\
\hline $\mathrm{H}$ & 22.11161500 & 16.80885100 & 25.44924200 \\
\hline $\mathrm{H}$ & 22.81979400 & 18.35578700 & 24.98414500 \\
\hline $\mathrm{H}$ & 19.81328700 & 17.82813600 & 25.24495400 \\
\hline $\mathrm{H}$ & 20.53185900 & 19.32459800 & 24.66202100 \\
\hline $\mathrm{H}$ & 21.36654500 & 19.93827500 & 26.73471500 \\
\hline $\mathrm{H}$ & 19.68768000 & 16.75216400 & 28.60438800 \\
\hline $\mathrm{H}$ & 19.92732100 & 16.59201500 & 26.93757400 \\
\hline $\mathrm{H}$ & 20.85386500 & 18.45832800 & 29.78356800 \\
\hline $\mathrm{H}$ & 21.08099600 & 20.00786500 & 28.94682300 \\
\hline $\mathrm{H}$ & 23.05374200 & 16.42581500 & 21.77106000 \\
\hline $\mathrm{H}$ & 26.25693800 & 10.61762100 & 31.15191900 \\
\hline $\mathrm{H}$ & 26.01609700 & 9.53714800 & 29.79160500 \\
\hline $\mathrm{H}$ & 23.85086400 & 10.31618600 & 31.82551000 \\
\hline $\mathrm{H}$ & 24.04190200 & 10.96902700 & 30.17838100 \\
\hline $\mathrm{H}$ & 23.97063600 & 9.31916300 & 28.36769400 \\
\hline $\mathrm{H}$ & 22.84542500 & 8.01189600 & 28.57622700 \\
\hline $\mathrm{H}$ & 25.50532600 & 7.64092100 & 31.39083000 \\
\hline $\mathrm{H}$ & 21.96322200 & 23.98088800 & 29.57058200 \\
\hline $\mathrm{H}$ & 22.88034300 & 25.22027400 & 28.70473800 \\
\hline $\mathrm{H}$ & 23.92170900 & 23.05059100 & 28.32135700 \\
\hline $\mathrm{H}$ & 23.17783100 & 23.53280800 & 26.79335900 \\
\hline $\mathrm{H}$ & 21.15855300 & 25.16731900 & 26.84381500 \\
\hline $\mathrm{H}$ & 20.94877900 & 8.21634500 & 22.23058800 \\
\hline $\mathrm{H}$ & 21.81878700 & 14.16252700 & 21.95221600 \\
\hline $\mathrm{H}$ & 20.77942200 & 15.38188400 & 21.20163900 \\
\hline $\mathrm{H}$ & 21.43019100 & 14.05284100 & 20.22459700 \\
\hline $\mathrm{H}$ & 20.72057200 & 14.46300300 & 24.32497700 \\
\hline
\end{tabular}




$\begin{array}{lrrr}\mathrm{H} & 18.29333000 & 14.85711500 & 20.79961900 \\ \mathrm{H} & 17.80339700 & 13.16964900 & 20.61553300 \\ \mathrm{H} & 18.98072600 & 13.89486100 & 19.50429200 \\ \mathrm{H} & 20.02268900 & 12.30679100 & 18.81600000 \\ \mathrm{H} & 18.45185100 & 11.53773500 & 19.04097500 \\ \mathrm{H} & 19.81734500 & 10.61350500 & 18.43207900 \\ \mathrm{H} & 22.05167000 & 8.35218500 & 25.64601100 \\ \mathrm{H} & 20.75389300 & 7.92710100 & 24.48810800 \\ \mathrm{H} & 20.90897500 & 14.19179900 & 26.48547800 \\ \mathrm{H} & 19.93009800 & 10.56500200 & 27.09736900 \\ \mathrm{H} & 20.40692900 & 9.46846700 & 29.26347300 \\ \mathrm{H} & 21.25706700 & 10.76277400 & 31.15956800 \\ \mathrm{H} & 21.68785200 & 13.21660200 & 30.90711300 \\ \mathrm{H} & 21.25749800 & 14.32020700 & 28.73053500 \\ \mathrm{H} & 25.73419200 & 8.73486900 & 32.75799600 \\ \mathrm{H} & 20.41903400 & 25.59409400 & 28.39791400 \\ \mathrm{H} & 20.24616700 & 23.95323900 & 27.75299100 \\ \mathrm{H} & 23.97252400 & 17.39396900 & 22.93229200 \\ \mathrm{H} & 23.39206900 & 15.77946300 & 23.38973000 \\ \mathrm{H} & 22.46245300 & 7.93463300 & 23.97440900 \\ \mathrm{H} & 27.10735200 & 8.32692100 & 31.71243000 \\ \mathrm{C} & 19.20667000 & 13.82817000 & 22.92852800 \\ \mathrm{H} & 18.27453900 & 13.26867800 & 23.05130600 \\ \mathrm{H} & 18.93927400 & 14.88860700 & 22.95324700 \\ \mathrm{C} & 19.98427300 & 8.57223900 & 19.78471400 \\ \mathrm{H} & 18.96940900 & 8.61569600 & 19.37350100 \\ \mathrm{H} & 20.67653900 & 8.64227200 & 18.93569900 \\ \mathrm{H} & 20.11679400 & 7.58064100 & 20.22681500 \\ \mathrm{H} & 23.72945400 & 12.59159500 & 26.33055500 \\ \mathrm{H} & 24.29953200 & 13.24908500 & 26.84529300 \\ & 18.57106300 & 11.88799500 & 25.33842000 \\ \mathrm{H} & 19.14488900 & 11.00194600 & 25.04881300 \\ \mathrm{H} & 18.08714500 & 11.69977400 & 26.29787200 \\ \mathrm{H} & 17.7583800 & 12.03472800 & 24.60647800\end{array}$

\section{Int2}

$\begin{array}{llll}\mathrm{C} & 23.14199700 & 16.68498200 & 22.83200000 \\ \mathrm{C} & 21.92142700 & 17.30279500 & 23.51657200 \\ \mathrm{C} & 22.23901600 & 17.80448300 & 24.92726300 \\ \mathrm{C} & 21.03692400 & 18.49361000 & 25.58540900 \\ \mathrm{~N} & 21.34521700 & 19.08351100 & 26.88411400 \\ \mathrm{C} & 21.53823600 & 18.39135600 & 27.99683300 \\ \mathrm{~N} & 21.16539000 & 17.08312400 & 28.09186100\end{array}$




\begin{tabular}{|c|c|c|c|}
\hline $\mathrm{N}$ & 22.07839300 & 18.99389700 & 29.06250300 \\
\hline $\mathrm{C}$ & 26.03298800 & 8.54201100 & 31.72098300 \\
\hline $\mathrm{C}$ & 26.07951300 & 9.51129900 & 30.53396900 \\
\hline $\mathrm{C}$ & 24.70767600 & 10.12208300 & 30.18568200 \\
\hline $\mathrm{C}$ & 23.68383100 & 9.05166300 & 29.81311800 \\
\hline $\mathrm{O}$ & 22.78314000 & 8.70600800 & 30.58607500 \\
\hline $\mathrm{N}$ & 23.88122100 & 8.48363800 & 28.59859900 \\
\hline $\mathrm{C}$ & 20.93700200 & 24.79702100 & 27.85201100 \\
\hline $\mathrm{C}$ & 22.32047500 & 24.55198300 & 28.46492400 \\
\hline $\mathrm{C}$ & 23.15665900 & 23.54011300 & 27.66439500 \\
\hline $\mathrm{C}$ & 22.59254200 & 22.11536800 & 27.77191200 \\
\hline $\mathrm{O}$ & 22.60385500 & 21.59688700 & 28.92688900 \\
\hline $\mathrm{O}$ & 22.15499400 & 21.58057300 & 26.71147000 \\
\hline $\mathrm{C}$ & 20.28873800 & 9.07871200 & 22.23917200 \\
\hline $\mathrm{C}$ & 19.46580200 & 9.55003700 & 21.20800400 \\
\hline $\mathrm{C}$ & 19.18335200 & 10.92448600 & 21.09843800 \\
\hline $\mathrm{C}$ & 22.57405300 & 10.36792500 & 24.84182400 \\
\hline $\mathrm{C}$ & 19.70727500 & 11.81986500 & 22.06593600 \\
\hline $\mathrm{C}$ & 24.42899500 & 10.67047400 & 26.18571800 \\
\hline $\mathrm{C}$ & 20.56183700 & 14.16356300 & 21.61697200 \\
\hline $\mathrm{C}$ & 19.40054600 & 13.32931800 & 22.20469700 \\
\hline $\mathrm{C}$ & 20.47830800 & 13.28242000 & 24.56915600 \\
\hline $\mathrm{O}$ & 25.31467500 & 10.28909700 & 26.98438300 \\
\hline $\mathrm{N}$ & 23.54142700 & 9.83017300 & 25.59588600 \\
\hline $\mathrm{C}$ & 23.35651100 & 12.65151000 & 25.10772100 \\
\hline $\mathrm{O}$ & 23.36653100 & 13.89395700 & 24.97122900 \\
\hline $\mathrm{C}$ & 22.39576600 & 11.72949100 & 24.60268500 \\
\hline $\mathrm{N}$ & 21.24486000 & 12.20824500 & 23.91424900 \\
\hline $\mathrm{C}$ & 18.07298100 & 13.81362100 & 21.58184400 \\
\hline $\mathrm{C}$ & 20.59860700 & 11.32758300 & 23.04349400 \\
\hline $\mathrm{C}$ & 18.39627600 & 11.39432800 & 19.88846400 \\
\hline $\mathrm{C}$ & 20.87175300 & 9.94415000 & 23.15915200 \\
\hline $\mathrm{N}$ & 21.67457400 & 9.47755200 & 24.22499600 \\
\hline $\mathrm{C}$ & 21.82278000 & 8.04300300 & 24.42626800 \\
\hline $\mathrm{O}$ & 18.67590100 & 15.96957300 & 26.40047500 \\
\hline $\mathrm{C}$ & 17.99839000 & 15.02363500 & 26.53795800 \\
\hline $\mathrm{O}$ & 17.27805300 & 14.11606300 & 26.67382000 \\
\hline $\mathrm{C}$ & 20.18010500 & 12.88016400 & 26.01117300 \\
\hline $\mathrm{C}$ & 20.76384200 & 13.56854900 & 27.00840100 \\
\hline $\mathrm{C}$ & 20.73033700 & 13.22874400 & 28.45190100 \\
\hline $\mathrm{C}$ & 21.25615400 & 12.00926600 & 28.91342500 \\
\hline $\mathrm{C}$ & 21.24923000 & 11.69225600 & 30.27353500 \\
\hline $\mathrm{C}$ & 20.72643500 & 12.60187100 & 31.19655400 \\
\hline $\mathrm{C}$ & 20.20412500 & 13.81940900 & 30.75587600 \\
\hline
\end{tabular}




\begin{tabular}{|c|c|c|c|}
\hline $\mathrm{C}$ & 20.20475200 & 14.12886200 & 29.39474400 \\
\hline $\mathrm{H}$ & 21.11825600 & 16.55562100 & 23.57245800 \\
\hline $\mathrm{H}$ & 21.52945800 & 18.13581400 & 22.91496700 \\
\hline $\mathrm{H}$ & 22.58058900 & 16.96157500 & 25.54150400 \\
\hline $\mathrm{H}$ & 23.06694500 & 18.52365400 & 24.89338900 \\
\hline $\mathrm{H}$ & 20.18533900 & 17.80691900 & 25.66204900 \\
\hline $\mathrm{H}$ & 20.70116400 & 19.32315900 & 24.95559100 \\
\hline $\mathrm{H}$ & 21.65668700 & 20.11204400 & 26.87568300 \\
\hline $\mathrm{H}$ & 21.60490100 & 16.49660300 & 28.78446100 \\
\hline $\mathrm{H}$ & 20.83677900 & 16.59642900 & 27.27259200 \\
\hline $\mathrm{H}$ & 21.98219300 & 18.54375500 & 29.95912800 \\
\hline $\mathrm{H}$ & 22.28438900 & 20.04301400 & 29.02582100 \\
\hline $\mathrm{H}$ & 22.90142800 & 16.36280600 & 21.81308800 \\
\hline $\mathrm{H}$ & 26.77941700 & 10.32831500 & 30.75202200 \\
\hline $\mathrm{H}$ & 26.46850500 & 9.00110700 & 29.64567100 \\
\hline $\mathrm{H}$ & 24.30236700 & 10.66711400 & 31.04330000 \\
\hline $\mathrm{H}$ & 24.83186300 & 10.80993600 & 29.34307600 \\
\hline $\mathrm{H}$ & 24.47206100 & 8.95366600 & 27.89952100 \\
\hline $\mathrm{H}$ & 23.15943200 & 7.85695900 & 28.27534800 \\
\hline $\mathrm{H}$ & 25.35022200 & 7.70966600 & 31.52341400 \\
\hline $\mathrm{H}$ & 22.21656800 & 24.16862100 & 29.48649200 \\
\hline $\mathrm{H}$ & 22.86283800 & 25.50477400 & 28.53292000 \\
\hline $\mathrm{H}$ & 24.17717600 & 23.51817900 & 28.06519200 \\
\hline $\mathrm{H}$ & 23.20593300 & 23.82759500 & 26.60904300 \\
\hline $\mathrm{H}$ & 21.02428100 & 25.21411000 & 26.84185400 \\
\hline $\mathrm{H}$ & 20.47179500 & 8.01317900 & 22.30836400 \\
\hline $\mathrm{H}$ & 21.51061200 & 13.92184000 & 22.10072000 \\
\hline $\mathrm{H}$ & 20.37048800 & 15.23498200 & 21.74996700 \\
\hline $\mathrm{H}$ & 20.66056800 & 13.96960600 & 20.54339700 \\
\hline $\mathrm{H}$ & 21.13686200 & 14.15438300 & 24.59450800 \\
\hline $\mathrm{H}$ & 17.85130500 & 14.81668100 & 21.96316500 \\
\hline $\mathrm{H}$ & 17.23777800 & 13.16118500 & 21.85502200 \\
\hline $\mathrm{H}$ & 18.10947300 & 13.89208900 & 20.49564700 \\
\hline $\mathrm{H}$ & 18.82236800 & 12.30631200 & 19.46542800 \\
\hline $\mathrm{H}$ & 17.34034900 & 11.59547000 & 20.10391500 \\
\hline $\mathrm{H}$ & 18.42040300 & 10.64616300 & 19.09481900 \\
\hline $\mathrm{H}$ & 22.41665800 & 7.89569800 & 25.32558700 \\
\hline $\mathrm{H}$ & 20.83653100 & 7.57993800 & 24.53988900 \\
\hline $\mathrm{H}$ & 21.39749800 & 14.40982300 & 26.72500600 \\
\hline $\mathrm{H}$ & 21.68229300 & 11.31361900 & 28.19587500 \\
\hline $\mathrm{H}$ & 21.65212500 & 10.73930600 & 30.60589500 \\
\hline $\mathrm{H}$ & 20.72456900 & 12.35863900 & 32.25554100 \\
\hline $\mathrm{H}$ & 19.78500400 & 14.52510000 & 31.46910200 \\
\hline $\mathrm{H}$ & 19.76849000 & 15.06540300 & 29.05643600 \\
\hline
\end{tabular}




$\begin{array}{lrrr}\mathrm{H} & 25.67289500 & 9.04684700 & 32.62502100 \\ \mathrm{H} & 20.34552400 & 25.49449400 & 28.45596300 \\ \mathrm{H} & 20.37693500 & 23.85958400 & 27.76896500 \\ \mathrm{H} & 23.96419800 & 17.40785900 & 22.77142800 \\ \mathrm{H} & 23.48638400 & 15.80665300 & 23.38831200 \\ \mathrm{H} & 22.34350000 & 7.56096900 & 23.58641000 \\ \mathrm{H} & 27.02447800 & 8.12754800 & 31.93573400 \\ \mathrm{C} & 19.24048000 & 13.60733200 & 23.72905500 \\ \mathrm{H} & 18.37264900 & 13.03588300 & 24.07487100 \\ \mathrm{H} & 19.00206700 & 14.66764000 & 23.87636300 \\ \mathrm{C} & 18.90618900 & 8.53422600 & 20.23222900 \\ \mathrm{H} & 17.81840200 & 8.61598800 & 20.12170100 \\ \mathrm{H} & 19.33692400 & 8.63557400 & 19.22706400 \\ \mathrm{H} & 19.12328200 & 7.51711900 & 20.57164100 \\ \mathrm{~N} & 24.34313000 & 12.02871600 & 25.88591300 \\ \mathrm{H} & 25.01986300 & 12.63822800 & 26.32433100 \\ \mathrm{C} & 19.36686600 & 11.62849700 & 26.22208400 \\ \mathrm{H} & 19.93717700 & 10.75346600 & 25.89159200 \\ \mathrm{H} & 19.10388300 & 11.48902900 & 27.27156700 \\ \mathrm{H} & 18.44246800 & 11.64529500 & 25.63653900\end{array}$

\section{Int2b}

$\begin{array}{lrrr}\mathrm{C} & 23.14201600 & 16.68500500 & 22.83201900 \\ \mathrm{C} & 21.66091800 & 17.05884100 & 22.92475600 \\ \mathrm{C} & 21.30805100 & 17.61847700 & 24.30466600 \\ \mathrm{C} & 19.87208900 & 18.14689500 & 24.39811400 \\ \mathrm{~N} & 19.62791000 & 18.82974000 & 25.67267800 \\ \mathrm{C} & 19.53478800 & 18.20861900 & 26.84116100 \\ \mathrm{~N} & 19.09627100 & 16.92152700 & 26.93213900 \\ \mathrm{~N} & 19.84368700 & 18.86910900 & 27.96382600 \\ \mathrm{C} & 26.03296500 & 8.54203600 & 31.72095000 \\ \mathrm{C} & 26.11640200 & 9.41336200 & 30.46036500 \\ \mathrm{C} & 24.94015900 & 10.40217200 & 30.30463600 \\ \mathrm{C} & 23.61273400 & 9.68454800 & 30.06363200 \\ \mathrm{O} & 22.83389300 & 9.41271900 & 30.98350100 \\ \mathrm{~N} & 23.40042800 & 9.32620000 & 28.77286000 \\ \mathrm{C} & 20.93700100 & 24.79701000 & 27.85200400 \\ \mathrm{C} & 22.16973300 & 23.90171300 & 27.68384200 \\ \mathrm{C} & 22.07069200 & 22.96336200 & 26.47710600 \\ \mathrm{C} & 21.11922000 & 21.76127200 & 26.64210100 \\ \mathrm{O} & 20.89106400 & 21.34648400 & 27.81558000 \\ \mathrm{O} & 20.68758700 & 21.25229800 & 25.56736500 \\ \mathrm{C} & 20.36902900 & 8.57954100 & 22.01452400\end{array}$




\begin{tabular}{|c|c|c|c|}
\hline $\mathrm{C}$ & 19.60766100 & 8.79832200 & 20.85899700 \\
\hline $\mathrm{C}$ & 19.04696200 & 10.06496800 & 20.60728800 \\
\hline $\mathrm{C}$ & 21.98487900 & 10.49755000 & 24.72240400 \\
\hline $\mathrm{C}$ & 19.22761700 & 11.10404400 & 21.55483300 \\
\hline $\mathrm{C}$ & 23.63219900 & 11.29792400 & 26.13672500 \\
\hline $\mathrm{C}$ & 19.58174400 & 13.56602400 & 21.04246800 \\
\hline $\mathrm{C}$ & 18.57391600 & 12.50587000 & 21.54483000 \\
\hline $\mathrm{C}$ & 19.33534600 & 12.84730500 & 23.99272100 \\
\hline $\mathrm{O}$ & 24.53680900 & 11.17512000 & 26.98949400 \\
\hline $\mathrm{N}$ & 22.97460000 & 10.24210200 & 25.58747800 \\
\hline $\mathrm{C}$ & 22.23868300 & 12.91008600 & 24.83329300 \\
\hline $\mathrm{O}$ & 21.98958100 & 14.10809100 & 24.57745000 \\
\hline $\mathrm{C}$ & 21.53748400 & 11.76604200 & 24.35664600 \\
\hline $\mathrm{N}$ & 20.38806900 & 11.92484600 & 23.53258700 \\
\hline $\mathrm{C}$ & 17.26383500 & 12.63826700 & 20.73895800 \\
\hline $\mathrm{C}$ & 20.06804600 & 10.87738400 & 22.66606900 \\
\hline $\mathrm{C}$ & 18.34646800 & 10.28383900 & 19.27845100 \\
\hline $\mathrm{C}$ & 20.62118600 & 9.60146800 & 22.92451200 \\
\hline $\mathrm{N}$ & 21.36169000 & 9.38998200 & 24.11246200 \\
\hline $\mathrm{C}$ & 21.82273000 & 8.04301100 & 24.42626800 \\
\hline $\mathrm{C}$ & 19.00150000 & 12.49737100 & 25.44139700 \\
\hline $\mathrm{C}$ & 19.40523300 & 13.34379400 & 26.41134200 \\
\hline $\mathrm{C}$ & 19.47042900 & 13.05721200 & 27.86524800 \\
\hline $\mathrm{C}$ & 20.31082600 & 12.03148300 & 28.33537300 \\
\hline $\mathrm{C}$ & 20.44005800 & 11.78323800 & 29.70303400 \\
\hline $\mathrm{C}$ & 19.73111000 & 12.56473000 & 30.61995100 \\
\hline $\mathrm{C}$ & 18.89625300 & 13.58867200 & 30.16762700 \\
\hline $\mathrm{C}$ & 18.76836000 & 13.83502500 & 28.79929000 \\
\hline $\mathrm{H}$ & 21.05338800 & 16.16689000 & 22.72755900 \\
\hline $\mathrm{H}$ & 21.40849800 & 17.80046900 & 22.15242200 \\
\hline $\mathrm{H}$ & 21.46418200 & 16.83241000 & 25.05366600 \\
\hline $\mathrm{H}$ & 21.98183300 & 18.44824700 & 24.55582600 \\
\hline $\mathrm{H}$ & 19.13806000 & 17.35513900 & 24.22828300 \\
\hline $\mathrm{H}$ & 19.70222500 & 18.90052800 & 23.62269700 \\
\hline $\mathrm{H}$ & 19.97655300 & 19.83842200 & 25.69902800 \\
\hline $\mathrm{H}$ & 19.43917900 & 16.34638900 & 27.68630600 \\
\hline $\mathrm{H}$ & 18.78177200 & 16.43530300 & 26.10040200 \\
\hline $\mathrm{H}$ & 19.49281500 & 18.51132000 & 28.83801500 \\
\hline $\mathrm{H}$ & 20.23453200 & 19.85726300 & 27.91867600 \\
\hline $\mathrm{H}$ & 23.39800100 & 16.31312300 & 21.83343100 \\
\hline $\mathrm{H}$ & 27.05282100 & 9.98590500 & 30.47243000 \\
\hline $\mathrm{H}$ & 26.15535900 & 8.77724600 & 29.56787000 \\
\hline $\mathrm{H}$ & 24.82432700 & 10.99413600 & 31.21810300 \\
\hline $\mathrm{H}$ & 25.13947200 & 11.07702700 & 29.46526600 \\
\hline
\end{tabular}




\begin{tabular}{|c|c|c|c|}
\hline $\mathrm{H}$ & 23.90629700 & 9.79904700 & 28.01279500 \\
\hline $\mathrm{H}$ & 22.50657100 & 8.90975100 & 28.55733000 \\
\hline $\mathrm{H}$ & 25.08770500 & 7.99155200 & 31.75207600 \\
\hline $\mathrm{H}$ & 22.29826800 & 23.28621500 & 28.58033000 \\
\hline $\mathrm{H}$ & 23.06527700 & 24.53095700 & 27.58630500 \\
\hline $\mathrm{H}$ & 23.05677000 & 22.52698700 & 26.26510300 \\
\hline $\mathrm{H}$ & 21.77923100 & 23.50486500 & 25.56983000 \\
\hline $\mathrm{H}$ & 20.78253900 & 25.42879500 & 26.96868700 \\
\hline $\mathrm{H}$ & 20.77107800 & 7.58868900 & 22.18830200 \\
\hline $\mathrm{H}$ & 20.50365400 & 13.54859100 & 21.62793000 \\
\hline $\mathrm{H}$ & 19.14839300 & 14.57124800 & 21.11522400 \\
\hline $\mathrm{H}$ & 19.83468900 & 13.38086000 & 19.99294300 \\
\hline $\mathrm{H}$ & 19.77059200 & 13.84921400 & 23.98533200 \\
\hline $\mathrm{H}$ & 16.77815400 & 13.58244900 & 21.01075000 \\
\hline $\mathrm{H}$ & 16.56558100 & 11.82736300 & 20.96941100 \\
\hline $\mathrm{H}$ & 17.41940000 & 12.66473000 & 19.66081100 \\
\hline $\mathrm{H}$ & 18.61130000 & 11.25029900 & 18.84517400 \\
\hline $\mathrm{H}$ & 17.25308400 & 10.24357200 & 19.34799900 \\
\hline $\mathrm{H}$ & 18.64584400 & 9.52723900 & 18.55158400 \\
\hline $\mathrm{H}$ & 22.32425100 & 8.07910600 & 25.39080000 \\
\hline $\mathrm{H}$ & 20.96796600 & 7.35949700 & 24.46552900 \\
\hline $\mathrm{H}$ & 19.86363600 & 14.27881100 & 26.08934100 \\
\hline $\mathrm{H}$ & 20.87969500 & 11.44632000 & 27.61825200 \\
\hline $\mathrm{H}$ & 21.09478200 & 10.99136400 & 30.05893400 \\
\hline $\mathrm{H}$ & 19.83500300 & 12.37542200 & 31.68450500 \\
\hline $\mathrm{H}$ & 18.33985900 & 14.19355900 & 30.87912300 \\
\hline $\mathrm{H}$ & 18.09772400 & 14.61694200 & 28.44796800 \\
\hline $\mathrm{H}$ & 26.07628300 & 9.16024700 & 32.62525500 \\
\hline $\mathrm{H}$ & 21.02853300 & 25.45401800 & 28.72464700 \\
\hline $\mathrm{H}$ & 20.04176900 & 24.18048900 & 27.98547300 \\
\hline $\mathrm{H}$ & 23.78140200 & 17.55096800 & 23.04272400 \\
\hline $\mathrm{H}$ & 23.36545100 & 15.89236600 & 23.55179200 \\
\hline $\mathrm{H}$ & 22.53958000 & 7.66962700 & 23.68108300 \\
\hline $\mathrm{H}$ & 26.85794700 & 7.82209000 & 31.76231100 \\
\hline $\mathrm{C}$ & 18.16117100 & 12.80471200 & 23.01581500 \\
\hline $\mathrm{H}$ & 17.42571800 & 12.04926300 & 23.31250300 \\
\hline $\mathrm{H}$ & 17.66090700 & 13.77616800 & 23.05585000 \\
\hline $\mathrm{C}$ & 19.42126100 & 7.63479100 & 19.90595400 \\
\hline $\mathrm{H}$ & 18.36726000 & 7.46177900 & 19.65836300 \\
\hline $\mathrm{H}$ & 19.95152200 & 7.78242800 & 18.95567300 \\
\hline $\mathrm{H}$ & 19.80798700 & 6.71164900 & 20.34757800 \\
\hline $\mathrm{N}$ & 23.27135800 & 12.58018100 & 25.72162800 \\
\hline $\mathrm{H}$ & 23.77529900 & 13.35314300 & 26.13462100 \\
\hline $\mathrm{C}$ & 18.43398600 & 11.12704000 & 25.71396100 \\
\hline
\end{tabular}




$\begin{array}{llll}\mathrm{H} & 19.13304700 & 10.35731400 & 25.36990200 \\ \mathrm{H} & 18.25000700 & 10.97080200 & 26.77819400 \\ \mathrm{H} & 17.49401000 & 10.96987800 & 25.17367900 \\ \mathrm{C} & 13.12105600 & 15.38295500 & 22.77302200 \\ \mathrm{C} & 14.49278200 & 14.89246900 & 23.25925600 \\ \mathrm{C} & 14.99800200 & 15.68178500 & 24.48600600 \\ \mathrm{C} & 16.36406600 & 15.21807600 & 24.94533800 \\ \mathrm{O} & 17.40112200 & 15.82249300 & 24.72110000 \\ \mathrm{H} & 14.44596100 & 13.82870500 & 23.51708500 \\ \mathrm{H} & 15.22714500 & 14.98521700 & 22.44996400 \\ \mathrm{H} & 15.07883200 & 16.74532700 & 24.24718300 \\ \mathrm{H} & 14.29084400 & 15.55996000 & 25.31426100 \\ \mathrm{H} & 13.15931700 & 16.43987900 & 22.48711200 \\ \mathrm{H} & 12.36355200 & 15.27653400 & 23.55771800 \\ \mathrm{H} & 12.78723400 & 14.81027900 & 21.90218100 \\ \mathrm{O} & 16.32226800 & 14.05069300 & 25.59721500 \\ \mathrm{H} & 17.25046800 & 13.76478700 & 25.78591700\end{array}$

\section{TS3}

C

C

C

C

$\mathrm{N}$

C

$\mathrm{N}$

$\mathrm{N}$

C

C

C

C

$\mathrm{O}$

$\mathrm{N}$

C

C

C

C

$\mathrm{O}$

$\mathrm{O}$

$\mathrm{C}$

C

C

C

16.68507200

17.01959800

17.60328600

18.13538800

18.86388300

18.29174500

16.96020400

19.06047500

8.54205700

9.60462400

10.08268400

8.95931100

8.26759200

8.75365800

24.79688300

23.80803100

22.89707600

21.76059600

21.42724400

21.22018500

8.64971800

8.89077000

10.17660400

10.54221900
22.83206600

22.87158300

24.22471600

24.24346400

25.48045900

26.64358100

26.74373200

27.75085400

31.72089000

30.62409900

30.41659300

29.90447400

30.66428800

28.56348900

27.85194100

27.77336800

26.54322500

26.59274600

27.72145300

25.48001200

21.86675600

20.64183000

20.33204100

24.72953100 


\begin{tabular}{|c|c|c|c|}
\hline $\mathrm{C}$ & 19.46828200 & 11.20414400 & 21.30046200 \\
\hline $\mathrm{C}$ & 23.33736400 & 11.35288900 & 26.31531200 \\
\hline $\mathrm{C}$ & 19.97531800 & 13.64959300 & 20.85624400 \\
\hline $\mathrm{C}$ & 18.86947300 & 12.62913300 & 21.21573000 \\
\hline $\mathrm{C}$ & 19.26368500 & 12.87364200 & 23.76749400 \\
\hline $\mathrm{O}$ & 24.14411200 & 11.22212200 & 27.25098300 \\
\hline $\mathrm{N}$ & 22.74766100 & 10.29442300 & 25.68468000 \\
\hline $\mathrm{C}$ & 22.12477600 & 12.97221200 & 24.85617300 \\
\hline $\mathrm{O}$ & 21.95453600 & 14.15682400 & 24.52610500 \\
\hline $\mathrm{C}$ & 21.40989200 & 11.82945700 & 24.35600900 \\
\hline $\mathrm{N}$ & 20.38560300 & 11.98538300 & 23.41168200 \\
\hline $\mathrm{C}$ & 17.68709400 & 12.81065600 & 20.23906100 \\
\hline $\mathrm{C}$ & 20.16468900 & 10.94538400 & 22.50204600 \\
\hline $\mathrm{C}$ & 18.85332600 & 10.41343600 & 18.92993000 \\
\hline $\mathrm{C}$ & 20.68093000 & 9.66781600 & 22.80129200 \\
\hline $\mathrm{N}$ & 21.33494300 & 9.45025200 & 24.03405500 \\
\hline $\mathrm{C}$ & 21.74405900 & 8.09564600 & 24.39384300 \\
\hline $\mathrm{C}$ & 18.69791700 & 12.34751100 & 25.12776700 \\
\hline $\mathrm{C}$ & 19.59023600 & 12.68860600 & 26.16587800 \\
\hline $\mathrm{C}$ & 19.99507800 & 12.04689200 & 27.39732900 \\
\hline $\mathrm{C}$ & 19.88961100 & 10.66280000 & 27.67660900 \\
\hline $\mathrm{C}$ & 20.39633300 & 10.13578500 & 28.85882800 \\
\hline $\mathrm{C}$ & 21.01170600 & 10.96171800 & 29.80378300 \\
\hline $\mathrm{C}$ & 21.13687400 & 12.32851500 & 29.54451000 \\
\hline $\mathrm{C}$ & 20.64563100 & 12.85928600 & 28.35833300 \\
\hline $\mathrm{H}$ & 21.07020400 & 16.10870200 & 22.67692000 \\
\hline $\mathrm{H}$ & 21.40469400 & 17.73556500 & 22.07264500 \\
\hline $\mathrm{H}$ & 21.35420300 & 16.83290300 & 24.99572200 \\
\hline $\mathrm{H}$ & 21.89834100 & 18.43833700 & 24.48875300 \\
\hline $\mathrm{H}$ & 19.06602800 & 17.33798500 & 24.08423400 \\
\hline $\mathrm{H}$ & 19.66123300 & 18.85881400 & 23.43242600 \\
\hline $\mathrm{H}$ & 19.91838200 & 19.84704800 & 25.52255800 \\
\hline $\mathrm{H}$ & 18.52590200 & 16.65802700 & 27.59610500 \\
\hline $\mathrm{H}$ & 18.66028200 & 16.43935400 & 25.91827300 \\
\hline $\mathrm{H}$ & 19.20670000 & 18.58377000 & 28.63929100 \\
\hline $\mathrm{H}$ & 19.68270500 & 20.01063600 & 27.72730100 \\
\hline $\mathrm{H}$ & 23.43525500 & 16.28027900 & 21.85649300 \\
\hline $\mathrm{H}$ & 26.51350300 & 10.47401200 & 30.86996000 \\
\hline $\mathrm{H}$ & 26.26717300 & 9.21293000 & 29.67173000 \\
\hline $\mathrm{H}$ & 24.01823900 & 10.42118600 & 31.36957200 \\
\hline $\mathrm{H}$ & 24.41973300 & 10.91444100 & 29.70502800 \\
\hline $\mathrm{H}$ & 23.89737600 & 9.52572500 & 27.95168800 \\
\hline $\mathrm{H}$ & 22.93288900 & 8.10376700 & 28.18820300 \\
\hline $\mathrm{H}$ & 25.39222800 & 7.67939700 & 31.51522700 \\
\hline
\end{tabular}




\begin{tabular}{|c|c|c|c|}
\hline $\mathrm{H}$ & 22.10297800 & 23.17303100 & 28.66559900 \\
\hline $\mathrm{H}$ & 23.05325900 & 24.36466800 & 27.76962200 \\
\hline $\mathrm{H}$ & 23.01318600 & 22.39764400 & 26.39945100 \\
\hline $\mathrm{H}$ & 21.87021400 & 23.47279000 & 25.62652000 \\
\hline $\mathrm{H}$ & 20.91398000 & 25.45379200 & 26.97360000 \\
\hline $\mathrm{H}$ & 20.87479500 & 7.65280700 & 22.07323500 \\
\hline $\mathrm{H}$ & 20.79389700 & 13.62724800 & 21.57908700 \\
\hline $\mathrm{H}$ & 19.56209800 & 14.66497900 & 20.84452700 \\
\hline $\mathrm{H}$ & 20.38328100 & 13.43685800 & 19.86193300 \\
\hline $\mathrm{H}$ & 19.67891400 & 13.86558300 & 23.94517300 \\
\hline $\mathrm{H}$ & 17.19494200 & 13.76302300 & 20.46429100 \\
\hline $\mathrm{H}$ & 16.94071100 & 12.01874000 & 20.35540400 \\
\hline $\mathrm{H}$ & 17.98970300 & 12.85224600 & 19.19272800 \\
\hline $\mathrm{H}$ & 19.18789600 & 11.37480900 & 18.53736200 \\
\hline $\mathrm{H}$ & 17.75937200 & 10.39708900 & 18.87097500 \\
\hline $\mathrm{H}$ & 19.22090300 & 9.65193300 & 18.24076300 \\
\hline $\mathrm{H}$ & 22.15025200 & 8.12949100 & 25.40194000 \\
\hline $\mathrm{H}$ & 20.87803400 & 7.42778300 & 24.35440300 \\
\hline $\mathrm{H}$ & 19.98629000 & 13.69901300 & 26.06812900 \\
\hline $\mathrm{H}$ & 19.45492200 & 9.99607900 & 26.94543100 \\
\hline $\mathrm{H}$ & 20.34495600 & 9.06796500 & 29.04813000 \\
\hline $\mathrm{H}$ & 21.41540700 & 10.52324700 & 30.70987100 \\
\hline $\mathrm{H}$ & 21.63615300 & 12.97524400 & 30.26034400 \\
\hline $\mathrm{H}$ & 20.76393700 & 13.91907100 & 28.14727500 \\
\hline $\mathrm{H}$ & 25.72838000 & 8.94420200 & 32.69421600 \\
\hline $\mathrm{H}$ & 21.00004500 & 25.42993500 & 28.74468300 \\
\hline $\mathrm{H}$ & 19.98978500 & 24.24912300 & 27.88989600 \\
\hline $\mathrm{H}$ & 23.75073200 & 17.57741500 & 23.02250000 \\
\hline $\mathrm{H}$ & 23.37223200 & 15.93088300 & 23.58937200 \\
\hline $\mathrm{H}$ & 22.52062400 & 7.71418300 & 23.71824900 \\
\hline $\mathrm{H}$ & 27.06865300 & 8.19491500 & 31.80690400 \\
\hline $\mathrm{C}$ & 18.26736800 & 12.94678600 & 22.61309600 \\
\hline $\mathrm{H}$ & 17.42861900 & 12.26419000 & 22.77665000 \\
\hline $\mathrm{H}$ & 17.85586000 & 13.95851200 & 22.60096100 \\
\hline $\mathrm{C}$ & 19.76471000 & 7.73562200 & 19.66792200 \\
\hline $\mathrm{H}$ & 18.74052600 & 7.60015500 & 19.30219900 \\
\hline $\mathrm{H}$ & 20.40398700 & 7.87008100 & 18.78576000 \\
\hline $\mathrm{H}$ & 20.06872700 & 6.79958500 & 20.14515600 \\
\hline $\mathrm{N}$ & 23.04763600 & 12.63619000 & 25.85136400 \\
\hline $\mathrm{H}$ & 23.52665000 & 13.40437000 & 26.30151500 \\
\hline $\mathrm{C}$ & 18.04290200 & 10.97131900 & 25.08536300 \\
\hline $\mathrm{H}$ & 18.75901200 & 10.17514000 & 24.85642900 \\
\hline $\mathrm{H}$ & 17.56917000 & 10.74464200 & 26.04444200 \\
\hline $\mathrm{H}$ & 17.25910900 & 10.94036200 & 24.32498300 \\
\hline
\end{tabular}




$\begin{array}{llll}\mathrm{C} & 13.19984700 & 15.33036200 & 22.80552500 \\ \mathrm{C} & 14.53981800 & 14.72064200 & 23.23939000 \\ \mathrm{C} & 15.30609100 & 15.59427900 & 24.24640600 \\ \mathrm{C} & 16.61025200 & 14.94363000 & 24.72076800 \\ \mathrm{O} & 17.69075800 & 15.56089400 & 24.62450600 \\ \mathrm{H} & 14.38250900 & 13.73645500 & 23.69242200 \\ \mathrm{H} & 15.17113300 & 14.55451700 & 22.35624500 \\ \mathrm{H} & 15.54678000 & 16.57339900 & 23.82125000 \\ \mathrm{H} & 14.67459300 & 15.75984400 & 25.12985200 \\ \mathrm{H} & 13.34296000 & 16.30991200 & 22.33470300 \\ \mathrm{H} & 12.53599600 & 15.47255900 & 23.66648600 \\ \mathrm{H} & 12.68290600 & 14.68547500 & 22.08661000 \\ \mathrm{O} & 16.48090800 & 13.74769700 & 25.17882800 \\ \mathrm{H} & 17.67600900 & 13.16442600 & 25.26418100\end{array}$

\section{Int3}

$\begin{array}{lrrr}\mathrm{C} & 23.14202300 & 16.68498800 & 22.83201000 \\ \mathrm{C} & 21.72029700 & 17.24899600 & 22.82449900 \\ \mathrm{C} & 21.30493700 & 17.77497400 & 24.19923100 \\ \mathrm{C} & 19.96437200 & 18.51790800 & 24.17400600 \\ \mathrm{~N} & 19.65256100 & 19.10918600 & 25.46967200 \\ \mathrm{C} & 19.08258400 & 18.45224000 & 26.47445300 \\ \mathrm{~N} & 18.70058300 & 17.15996800 & 26.36953600 \\ \mathrm{~N} & 18.88444000 & 19.11986000 & 27.63458800 \\ \mathrm{C} & 26.03296300 & 8.54203400 & 31.72095200 \\ \mathrm{C} & 26.15446300 & 9.46408200 & 30.50352100 \\ \mathrm{C} & 24.84756100 & 10.21580100 & 30.17431300 \\ \mathrm{C} & 23.73622900 & 9.25399100 & 29.76625200 \\ \mathrm{O} & 22.95702100 & 8.76816200 & 30.58832500 \\ \mathrm{~N} & 23.72615600 & 8.92274900 & 28.44541300 \\ \mathrm{C} & 20.93699800 & 24.79704000 & 27.85201500 \\ \mathrm{C} & 21.91536100 & 23.78589900 & 28.45914200 \\ \mathrm{C} & 22.32666100 & 22.69279900 & 27.46858400 \\ \mathrm{C} & 21.21079600 & 21.68196200 & 27.12277400 \\ \mathrm{O} & 20.36553800 & 21.42076300 & 28.02490100 \\ \mathrm{O} & 21.27308700 & 21.16709000 & 25.96926400 \\ \mathrm{C} & 19.75513100 & 8.54152100 & 22.54493700 \\ \mathrm{C} & 18.73803500 & 8.72250900 & 21.61254400 \\ \mathrm{C} & 18.05810500 & 9.95860500 & 21.55926200 \\ \mathrm{C} & 21.79328600 & 10.40792600 & 24.96779500 \\ \mathrm{C} & 18.36128700 & 10.97921700 & 22.48474600 \\ \mathrm{C} & 23.61899300 & 11.32697600 & 26.06528200 \\ \mathrm{C} & 18.18161500 & 13.41757800 & 21.82187900\end{array}$




\begin{tabular}{|c|c|c|c|}
\hline $\mathrm{C}$ & 17.55694300 & 12.28574400 & 22.67470700 \\
\hline $\mathrm{C}$ & 19.05328100 & 12.94332400 & 24.60474800 \\
\hline $\mathrm{O}$ & 24.58407500 & 11.24800200 & 26.82302300 \\
\hline $\mathrm{N}$ & 22.91072800 & 10.23537300 & 25.63497500 \\
\hline $\mathrm{C}$ & 22.09854800 & 12.85387400 & 24.85597700 \\
\hline $\mathrm{O}$ & 21.87982900 & 13.90445500 & 24.28758600 \\
\hline $\mathrm{C}$ & 21.05697800 & 11.72167600 & 25.02859300 \\
\hline $\mathrm{N}$ & 19.95491300 & 11.87070700 & 24.11371500 \\
\hline $\mathrm{C}$ & 16.04246400 & 12.16891200 & 22.38388400 \\
\hline $\mathrm{C}$ & 19.48929100 & 10.81772500 & 23.33898300 \\
\hline $\mathrm{C}$ & 17.05247500 & 10.15429800 & 20.44077300 \\
\hline $\mathrm{C}$ & 20.15350400 & 9.57447300 & 23.38898300 \\
\hline $\mathrm{N}$ & 21.24054800 & 9.38356400 & 24.29233600 \\
\hline $\mathrm{C}$ & 21.82274100 & 8.04299800 & 24.42626400 \\
\hline $\mathrm{C}$ & 19.36436200 & 13.07427900 & 26.13501700 \\
\hline $\mathrm{C}$ & 20.39493500 & 11.95419300 & 26.46976200 \\
\hline $\mathrm{C}$ & 19.89908400 & 10.65408800 & 27.06937700 \\
\hline $\mathrm{C}$ & 18.85423700 & 9.90026600 & 26.51315200 \\
\hline $\mathrm{C}$ & 18.50785000 & 8.65878000 & 27.04601900 \\
\hline $\mathrm{C}$ & 19.19411300 & 8.14655000 & 28.14944400 \\
\hline $\mathrm{C}$ & 20.22754400 & 8.88818700 & 28.72464700 \\
\hline $\mathrm{C}$ & 20.57003400 & 10.12836400 & 28.18211000 \\
\hline $\mathrm{H}$ & 21.01235700 & 16.47363700 & 22.50575500 \\
\hline $\mathrm{H}$ & 21.65030900 & 18.06181500 & 22.08692200 \\
\hline $\mathrm{H}$ & 21.24091200 & 16.93854600 & 24.90646800 \\
\hline $\mathrm{H}$ & 22.06593100 & 18.46601900 & 24.58585100 \\
\hline $\mathrm{H}$ & 19.15489000 & 17.85893600 & 23.84487500 \\
\hline $\mathrm{H}$ & 20.02211800 & 19.34814000 & 23.46084500 \\
\hline $\mathrm{H}$ & 20.20971700 & 19.97974500 & 25.70998300 \\
\hline $\mathrm{H}$ & 17.90490700 & 16.86510000 & 26.92377700 \\
\hline $\mathrm{H}$ & 18.61054500 & 16.72051800 & 25.43241900 \\
\hline $\mathrm{H}$ & 18.74460000 & 18.54864000 & 28.45367200 \\
\hline $\mathrm{H}$ & 19.38889300 & 20.02913000 & 27.77602600 \\
\hline $\mathrm{H}$ & 23.43848800 & 16.32348700 & 21.84018900 \\
\hline $\mathrm{H}$ & 26.94649500 & 10.20348800 & 30.67663700 \\
\hline $\mathrm{H}$ & 26.45822700 & 8.88454100 & 29.62287700 \\
\hline $\mathrm{H}$ & 24.49512500 & 10.75430800 & 31.05935000 \\
\hline $\mathrm{H}$ & 25.02796800 & 10.93920200 & 29.37186900 \\
\hline $\mathrm{H}$ & 24.17793400 & 9.53394500 & 27.76954800 \\
\hline $\mathrm{H}$ & 22.91769200 & 8.41395000 & 28.11765700 \\
\hline $\mathrm{H}$ & 25.23073600 & 7.81156500 & 31.58394800 \\
\hline $\mathrm{H}$ & 21.44603600 & 23.29874200 & 29.32043200 \\
\hline $\mathrm{H}$ & 22.80883900 & 24.31284300 & 28.82324200 \\
\hline $\mathrm{H}$ & 23.15085700 & 22.10182700 & 27.89265100 \\
\hline
\end{tabular}




\begin{tabular}{|c|c|c|c|}
\hline $\mathrm{H}$ & 22.70273400 & 23.12193700 & 26.53295300 \\
\hline $\mathrm{H}$ & 21.37291000 & 25.29454200 & 26.97682800 \\
\hline $\mathrm{H}$ & 20.25500600 & 7.58233300 & 22.58292500 \\
\hline $\mathrm{H}$ & 19.27229500 & 13.41216400 & 21.90149300 \\
\hline $\mathrm{H}$ & 17.84047000 & 14.39979400 & 22.16232400 \\
\hline $\mathrm{H}$ & 17.92597400 & 13.28596800 & 20.76472500 \\
\hline $\mathrm{H}$ & 19.33647700 & 13.88647600 & 24.13804200 \\
\hline $\mathrm{H}$ & 15.53231000 & 13.02386500 & 22.83358600 \\
\hline $\mathrm{H}$ & 15.62223700 & 11.25938500 & 22.82676400 \\
\hline $\mathrm{H}$ & 15.79611800 & 12.18023500 & 21.32311300 \\
\hline $\mathrm{H}$ & 17.03907400 & 11.18613400 & 20.09213600 \\
\hline $\mathrm{H}$ & 16.03064800 & 9.88721200 & 20.73343700 \\
\hline $\mathrm{H}$ & 17.31216700 & 9.53419800 & 19.58032300 \\
\hline $\mathrm{H}$ & 22.56797200 & 8.07816600 & 25.21577100 \\
\hline $\mathrm{H}$ & 21.02884600 & 7.33875900 & 24.68539500 \\
\hline $\mathrm{H}$ & 21.15037300 & 12.35295300 & 27.15156500 \\
\hline $\mathrm{H}$ & 18.31830600 & 10.27009700 & 25.64885100 \\
\hline $\mathrm{H}$ & 17.69843400 & 8.09225000 & 26.59364100 \\
\hline $\mathrm{H}$ & 18.92217000 & 7.17843000 & 28.56120600 \\
\hline $\mathrm{H}$ & 20.79011300 & 8.51561000 & 29.57633600 \\
\hline $\mathrm{H}$ & 21.38996600 & 10.68580600 & 28.62505700 \\
\hline $\mathrm{H}$ & 25.79328700 & 9.11813700 & 32.62191200 \\
\hline $\mathrm{H}$ & 20.65345000 & 25.57215100 & 28.57381900 \\
\hline $\mathrm{H}$ & 20.02449200 & 24.28549200 & 27.52908800 \\
\hline $\mathrm{H}$ & 23.86501400 & 17.45061600 & 23.13875000 \\
\hline $\mathrm{H}$ & 23.21412800 & 15.84310300 & 23.52664300 \\
\hline $\mathrm{H}$ & 22.30120300 & 7.73471800 & 23.49227000 \\
\hline $\mathrm{H}$ & 26.96878000 & 8.00240800 & 31.90337900 \\
\hline $\mathrm{C}$ & 17.63727600 & 12.62634600 & 24.18740800 \\
\hline $\mathrm{H}$ & 17.23030500 & 11.77985100 & 24.75291500 \\
\hline $\mathrm{H}$ & 17.01526400 & 13.48888400 & 24.41920500 \\
\hline $\mathrm{C}$ & 18.39626200 & 7.57880400 & 20.68239900 \\
\hline $\mathrm{H}$ & 17.32325900 & 7.35580900 & 20.67780800 \\
\hline $\mathrm{H}$ & 18.68182000 & 7.78669300 & 19.64290500 \\
\hline $\mathrm{H}$ & 18.91991200 & 6.66670600 & 20.98384800 \\
\hline $\mathrm{N}$ & 23.27373800 & 12.58537400 & 25.52938300 \\
\hline $\mathrm{H}$ & 23.93480200 & 13.34118600 & 25.66003700 \\
\hline $\mathrm{C}$ & 18.15067100 & 13.15296700 & 27.06244900 \\
\hline $\mathrm{H}$ & 17.54464000 & 12.24415300 & 27.03565100 \\
\hline $\mathrm{H}$ & 18.48224500 & 13.29194800 & 28.09737100 \\
\hline $\mathrm{H}$ & 17.52371600 & 14.00394300 & 26.77660200 \\
\hline $\mathrm{C}$ & 13.12104300 & 15.38295700 & 22.77302200 \\
\hline $\mathrm{C}$ & 14.17946000 & 15.40083600 & 23.88017200 \\
\hline $\mathrm{C}$ & 15.53510100 & 15.91790800 & 23.39869100 \\
\hline
\end{tabular}


O

$\mathrm{H}$

$\mathrm{H}$

$\mathrm{H}$

$\mathrm{H}$

$\mathrm{H}$

$\mathrm{H}$

$\mathrm{H}$

$\mathrm{O}$

$\mathrm{H}$

TS4

C

C

C

C

$\mathrm{N}$

C

$\mathrm{N}$

$\mathrm{N}$

C

C

C

C

$\mathrm{O}$

$\mathrm{N}$

C

C

C

C

O

$\mathrm{O}$

C

C

C

C

C

C

C

C

C

$\mathrm{O}$
16.65717800

17.84397000

13.84829800

14.30791200

15.86515900

15.45330700

13.43089400

12.96084400

12.15486800

16.32564500

19.90294700
15.84543400

15.99948100

16.01170100

14.39174600

15.36912100

16.96599900

14.73633400

16.38662700

15.01515400

15.64280800

14.02058100
24.45715000

24.01639200

24.72629300

24.29083000

22.50803700

23.07770600

21.94241400

22.36021500

23.13813700

25.65132700

26.24494900

$\begin{array}{rrr}23.14201500 & 16.68499000 & 22.83201300 \\ 21.86766400 & 17.53187300 & 22.81871700 \\ 21.43955300 & 17.96210000 & 24.22197000 \\ 20.18729400 & 18.84774000 & 24.21516100 \\ 19.84727400 & 19.32529200 & 25.54976500 \\ 19.22897900 & 18.58097100 & 26.46338800 \\ 18.79719300 & 17.32961500 & 26.18858200 \\ 19.04032000 & 19.11008600 & 27.68968800 \\ 26.03295000 & 8.54204200 & 31.72092800 \\ 26.09749600 & 9.43902000 & 30.48088700 \\ 24.71900700 & 9.69286600 & 29.83556900 \\ 24.09822300 & 8.40177900 & 29.31029800 \\ 23.35067000 & 7.70693900 & 30.00497300 \\ 24.48043000 & 8.05077200 & 28.05634400 \\ 20.93699600 & 24.79702800 & 27.85201300 \\ 21.78746400 & 23.89405200 & 28.75205900 \\ 22.41072600 & 22.72000400 & 27.98777200 \\ 21.37276300 & 21.68714700 & 27.50263700 \\ 20.49074000 & 21.33724100 & 28.33853700 \\ 21.50876300 & 21.26085100 & 26.32025000 \\ 20.09049000 & 9.02425800 & 22.36415100 \\ 19.23796800 & 9.47819500 & 21.36186600 \\ 18.96306400 & 10.85852300 & 21.22797300 \\ 22.51167500 & 10.37691300 & 24.76450600 \\ 19.46796100 & 11.76670500 & 22.18319600 \\ 24.45031400 & 10.74624900 & 25.97858400 \\ 20.23629300 & 14.08144900 & 21.51826000 \\ 19.14578600 & 13.27578300 & 22.25778400 \\ 20.40075100 & 13.34034500 & 24.46729700 \\ 25.42042300 & 10.37505900 & 26.65582500\end{array}$




\begin{tabular}{|c|c|c|c|}
\hline $\mathrm{N}$ & 23.55918900 & 9.88699900 & 25.41180300 \\
\hline $\mathrm{C}$ & 23.19032200 & 12.71832700 & 25.12214400 \\
\hline $\mathrm{O}$ & 23.15059000 & 13.94628300 & 25.00892800 \\
\hline $\mathrm{C}$ & 22.19308000 & 11.76025000 & 24.67306700 \\
\hline $\mathrm{N}$ & 21.01533300 & 12.17285200 & 24.05260200 \\
\hline $\mathrm{C}$ & 17.75872800 & 13.71319100 & 21.73435200 \\
\hline $\mathrm{C}$ & 20.36409900 & 11.27711900 & 23.16459500 \\
\hline $\mathrm{C}$ & 18.17019900 & 11.30822100 & 20.01646800 \\
\hline $\mathrm{C}$ & 20.70416000 & 9.91477300 & 23.24501000 \\
\hline $\mathrm{N}$ & 21.63695300 & 9.47437300 & 24.19627600 \\
\hline $\mathrm{C}$ & 21.82272900 & 8.04301200 & 24.42628100 \\
\hline $\mathrm{C}$ & 20.15283700 & 13.26820200 & 26.46740600 \\
\hline $\mathrm{C}$ & 20.90280000 & 12.16741800 & 26.95195700 \\
\hline $\mathrm{C}$ & 20.47193300 & 10.79131600 & 27.07948500 \\
\hline $\mathrm{C}$ & 19.40172100 & 10.24435000 & 26.33417100 \\
\hline $\mathrm{C}$ & 19.04154800 & 8.90830500 & 26.47452700 \\
\hline $\mathrm{C}$ & 19.73814800 & 8.07601600 & 27.35619900 \\
\hline $\mathrm{C}$ & 20.81915800 & 8.58509900 & 28.08397700 \\
\hline $\mathrm{C}$ & 21.18489000 & 9.91844900 & 27.93465300 \\
\hline $\mathrm{H}$ & 21.04867700 & 16.96946900 & 22.35226400 \\
\hline $\mathrm{H}$ & 22.01989100 & 18.42437500 & 22.19486600 \\
\hline $\mathrm{H}$ & 21.24815500 & 17.07435100 & 24.83824300 \\
\hline $\mathrm{H}$ & 22.25065500 & 18.51632600 & 24.71155500 \\
\hline $\mathrm{H}$ & 19.33800100 & 18.31946200 & 23.76900300 \\
\hline $\mathrm{H}$ & 20.37243300 & 19.73931100 & 23.60613900 \\
\hline $\mathrm{H}$ & 20.43146500 & 20.13996400 & 25.89969800 \\
\hline $\mathrm{H}$ & 18.02135700 & 16.96647900 & 26.72934400 \\
\hline $\mathrm{H}$ & 18.65496200 & 17.04302100 & 25.20189100 \\
\hline $\mathrm{H}$ & 18.81200900 & 18.46627100 & 28.43061900 \\
\hline $\mathrm{H}$ & 19.55860400 & 19.98992800 & 27.94674900 \\
\hline $\mathrm{H}$ & 23.43190100 & 16.37968300 & 21.81945700 \\
\hline $\mathrm{H}$ & 26.53946000 & 10.40745900 & 30.74669300 \\
\hline $\mathrm{H}$ & 26.75827700 & 8.99419800 & 29.72691600 \\
\hline $\mathrm{H}$ & 24.02713900 & 10.09823100 & 30.58069800 \\
\hline $\mathrm{H}$ & 24.83304900 & 10.41228000 & 29.01898800 \\
\hline $\mathrm{H}$ & 24.90682900 & 8.73485900 & 27.42681800 \\
\hline $\mathrm{H}$ & 24.01978100 & 7.24722400 & 27.65634700 \\
\hline $\mathrm{H}$ & 25.56981400 & 7.57939400 & 31.48654100 \\
\hline $\mathrm{H}$ & 21.16310600 & 23.47965000 & 29.55095900 \\
\hline $\mathrm{H}$ & 22.57842500 & 24.49049900 & 29.22823800 \\
\hline $\mathrm{H}$ & 23.10565000 & 22.18352000 & 28.64829000 \\
\hline $\mathrm{H}$ & 22.98629500 & 23.07041000 & 27.12426000 \\
\hline $\mathrm{H}$ & 21.53558000 & 25.21737200 & 27.03474300 \\
\hline $\mathrm{H}$ & 20.30339400 & 7.96518300 & 22.43121700 \\
\hline
\end{tabular}




\begin{tabular}{|c|c|c|c|}
\hline $\mathrm{H}$ & 21.23302800 & 13.89676100 & 21.92926600 \\
\hline $\mathrm{H}$ & 20.02169000 & 15.15045000 & 21.60742900 \\
\hline $\mathrm{H}$ & 20.25579500 & 13.82193100 & 20.45433700 \\
\hline $\mathrm{H}$ & 21.10385700 & 14.15434400 & 24.57203300 \\
\hline $\mathrm{H}$ & 17.57280800 & 14.72418700 & 22.10407600 \\
\hline $\mathrm{H}$ & 16.96373000 & 13.06004900 & 22.10873700 \\
\hline $\mathrm{H}$ & 17.69752800 & 13.74828500 & 20.64716400 \\
\hline $\mathrm{H}$ & 18.55701100 & 12.24439800 & 19.61415700 \\
\hline $\mathrm{H}$ & 17.10748300 & 11.45805600 & 20.23450900 \\
\hline $\mathrm{H}$ & 18.23538500 & 10.57100600 & 19.21485300 \\
\hline $\mathrm{H}$ & 22.48239300 & 7.93121400 & 25.28189800 \\
\hline $\mathrm{H}$ & 20.85285300 & 7.59139000 & 24.64785200 \\
\hline $\mathrm{H}$ & 21.87492100 & 12.38262400 & 27.38716900 \\
\hline $\mathrm{H}$ & 18.87807000 & 10.86190600 & 25.61582000 \\
\hline $\mathrm{H}$ & 18.21889100 & 8.51354400 & 25.88451100 \\
\hline $\mathrm{H}$ & 19.44720900 & 7.03534900 & 27.46987600 \\
\hline $\mathrm{H}$ & 21.38490000 & 7.95378300 & 28.76300700 \\
\hline $\mathrm{H}$ & 22.03160500 & 10.30403100 & 28.49562300 \\
\hline $\mathrm{H}$ & 25.42667600 & 9.00758500 & 32.50631500 \\
\hline $\mathrm{H}$ & 20.49775900 & 25.63076500 & 28.41235700 \\
\hline $\mathrm{H}$ & 20.11871100 & 24.22332700 & 27.40454200 \\
\hline $\mathrm{H}$ & 23.97851400 & 17.24917100 & 23.26110900 \\
\hline $\mathrm{H}$ & 23.01640900 & 15.78025300 & 23.43445600 \\
\hline $\mathrm{H}$ & 22.27577400 & 7.55533700 & 23.55573500 \\
\hline $\mathrm{H}$ & 27.03284800 & 8.35821400 & 32.12931400 \\
\hline $\mathrm{C}$ & 19.11419000 & 13.64267500 & 23.76473900 \\
\hline $\mathrm{H}$ & 18.28926900 & 13.07913600 & 24.21512000 \\
\hline $\mathrm{H}$ & 18.87535000 & 14.70159600 & 23.88608400 \\
\hline $\mathrm{C}$ & 18.63936800 & 8.45495700 & 20.42039800 \\
\hline $\mathrm{H}$ & 17.55400500 & 8.56936800 & 20.32850700 \\
\hline $\mathrm{H}$ & 19.05647200 & 8.53245800 & 19.40863700 \\
\hline $\mathrm{H}$ & 18.83691800 & 7.44022100 & 20.77649800 \\
\hline $\mathrm{N}$ & 24.26336600 & 12.11463900 & 25.77182400 \\
\hline $\mathrm{H}$ & 24.96116300 & 12.73917100 & 26.15500500 \\
\hline $\mathrm{C}$ & 18.65396100 & 13.40861200 & 26.73142600 \\
\hline $\mathrm{H}$ & 18.07118600 & 12.53212200 & 26.44982600 \\
\hline $\mathrm{H}$ & 18.51563700 & 13.54881900 & 27.81019700 \\
\hline $\mathrm{H}$ & 18.22014200 & 14.27812900 & 26.23120500 \\
\hline $\mathrm{C}$ & 13.12107800 & 15.38294500 & 22.77302800 \\
\hline $\mathrm{C}$ & 14.41964900 & 15.17154400 & 23.55939100 \\
\hline $\mathrm{C}$ & 15.43440600 & 16.30005000 & 23.36234000 \\
\hline $\mathrm{C}$ & 16.68982700 & 16.16130800 & 24.25013300 \\
\hline $\mathrm{O}$ & 17.79373300 & 16.53586300 & 23.72561300 \\
\hline $\mathrm{H}$ & 14.21652000 & 15.08532000 & 24.63154400 \\
\hline
\end{tabular}




$\begin{array}{llll}\mathrm{H} & 14.87967600 & 14.21906100 & 23.26194500 \\ \mathrm{H} & 15.74229300 & 16.38265000 & 22.31387300 \\ \mathrm{H} & 14.96321300 & 17.25950800 & 23.62326400 \\ \mathrm{H} & 13.31563100 & 15.45607100 & 21.69583700 \\ \mathrm{H} & 12.62457000 & 16.31234400 & 23.07769000 \\ \mathrm{H} & 12.41246900 & 14.56064500 & 22.92813700 \\ \mathrm{O} & 16.53626300 & 15.71498400 & 25.41230300 \\ \mathrm{H} & 20.66488800 & 14.20988800 & 26.66016900\end{array}$

\section{Prod}

C

C

C

C

$\mathrm{N}$

C

$\mathrm{N}$

$\mathrm{N}$

C

C

C

C

$\mathrm{O}$

$\mathrm{N}$

C

C

C

C

$\mathrm{O}$

$\mathrm{O}$

C

C

C

C

C

C

C

C

C

$\mathrm{O}$

$\mathrm{N}$

C

$\mathrm{O}$
23.14202400

21.83649300

21.39924300

20.13342700

19.76621700

19.19515100

18.78705300

19.03168200

26.03296300

26.28023200

24.98583100

24.24155700

23.41442000

24.60934600

20.93699900

21.73863600

22.33762800

21.28551600

20.44410000

21.37177300

19.94009600

19.05904200

18.90926700

22.64247100

19.56420800

24.66718900

20.50081400

19.39812100

20.79648800

25.65473200

23.69536300

23.46270800

23.45468500
16.68498800

17.48433000

17.93898000

18.80591500

19.27504900

18.50372900

17.24489300

19.01271400

8.54203400

9.31307800

9.69137300

8.45547800

7.86431200

8.02373000

24.79704400

23.83303000

22.66284500

21.64407500

21.25113600

21.26571500

9.06313600

9.51441300

10.89718800

10.35611100

11.82427200

10.61940600

13.92653000

13.35891500

13.45042400

10.20554000

9.81039200

12.64724100

13.88469100
22.83201000

22.80396500

24.19734100

24.17238700

25.50455100

26.42652000

26.14482800

27.66453200

31.72095200

30.41991500

29.66802400

29.17015400

29.87035700

27.93621300

27.85201600

28.73393000

27.94290400

27.45876200

28.31778600

26.25559600

22.55987800

21.58538800

21.33518000

24.68782600

22.17430800

25.78260700

21.16767000

22.09145500

24.17924200

26.41186400

25.28670800

24.98087400

24.91939000 


\begin{tabular}{|c|c|c|c|}
\hline $\mathrm{C}$ & 22.44611200 & 11.73954100 & 24.51522200 \\
\hline $\mathrm{N}$ & 21.23338200 & 12.23507500 & 23.96593500 \\
\hline $\mathrm{C}$ & 18.02201700 & 13.88382100 & 21.62746500 \\
\hline $\mathrm{C}$ & 20.48238700 & 11.32899300 & 23.13076900 \\
\hline $\mathrm{C}$ & 18.07921100 & 11.31209400 & 20.13615600 \\
\hline $\mathrm{C}$ & 20.71528600 & 9.95163000 & 23.31226300 \\
\hline $\mathrm{N}$ & 21.68522800 & 9.48022500 & 24.19208800 \\
\hline $\mathrm{C}$ & 21.82274900 & 8.04298900 & 24.42626500 \\
\hline $\mathrm{C}$ & 20.22068600 & 13.91276800 & 27.41264000 \\
\hline $\mathrm{C}$ & 20.99379700 & 12.81106600 & 27.37502700 \\
\hline $\mathrm{C}$ & 20.56515900 & 11.40128600 & 27.30389500 \\
\hline $\mathrm{C}$ & 19.46069600 & 10.98721500 & 26.53625700 \\
\hline $\mathrm{C}$ & 19.09126100 & 9.64352300 & 26.48262300 \\
\hline $\mathrm{C}$ & 19.81801500 & 8.68465700 & 27.19168500 \\
\hline $\mathrm{C}$ & 20.93485500 & 9.07168200 & 27.93832600 \\
\hline $\mathrm{C}$ & 21.30668400 & 10.41389000 & 27.97943600 \\
\hline $\mathrm{H}$ & 21.03386500 & 16.88191900 & 22.35696400 \\
\hline $\mathrm{H}$ & 21.95339500 & 18.36385400 & 22.15472600 \\
\hline $\mathrm{H}$ & 21.21986700 & 17.06184600 & 24.83210800 \\
\hline $\mathrm{H}$ & 22.20192700 & 18.51428200 & 24.67620900 \\
\hline $\mathrm{H}$ & 19.29914800 & 18.26883600 & 23.71204800 \\
\hline $\mathrm{H}$ & 20.31609500 & 19.70333900 & 23.57183000 \\
\hline $\mathrm{H}$ & 20.32088000 & 20.11154600 & 25.85125800 \\
\hline $\mathrm{H}$ & 18.12139100 & 16.82115200 & 26.77508900 \\
\hline $\mathrm{H}$ & 18.59727200 & 16.96269600 & 25.15927800 \\
\hline $\mathrm{H}$ & 18.82026200 & 18.36195900 & 28.40438000 \\
\hline $\mathrm{H}$ & 19.54180300 & 19.90008100 & 27.92259400 \\
\hline $\mathrm{H}$ & 23.44068700 & 16.37013900 & 21.82495700 \\
\hline $\mathrm{H}$ & 26.83638900 & 10.23400200 & 30.63630500 \\
\hline $\mathrm{H}$ & 26.91306700 & 8.72133800 & 29.74688500 \\
\hline $\mathrm{H}$ & 24.30665300 & 10.22361300 & 30.34206100 \\
\hline $\mathrm{H}$ & 25.23481400 & 10.34195400 & 28.82396200 \\
\hline $\mathrm{H}$ & 25.07863400 & 8.65600100 & 27.28161100 \\
\hline $\mathrm{H}$ & 24.06523600 & 7.26380300 & 27.55520800 \\
\hline $\mathrm{H}$ & 25.44415000 & 7.63966100 & 31.53434800 \\
\hline $\mathrm{H}$ & 21.08894900 & 23.41324300 & 29.50966300 \\
\hline $\mathrm{H}$ & 22.54030600 & 24.38647600 & 29.24282700 \\
\hline $\mathrm{H}$ & 23.03346600 & 22.10924400 & 28.58784600 \\
\hline $\mathrm{H}$ & 22.90628700 & 23.02023900 & 27.07777100 \\
\hline $\mathrm{H}$ & 21.56844200 & 25.23283900 & 27.06835300 \\
\hline $\mathrm{H}$ & 20.05341800 & 7.99771500 & 22.70596000 \\
\hline $\mathrm{H}$ & 21.50354300 & 13.68913300 & 21.53652100 \\
\hline $\mathrm{H}$ & 20.41242700 & 15.01560900 & 21.10639400 \\
\hline $\mathrm{H}$ & 20.40843100 & 13.51740600 & 20.15641300 \\
\hline
\end{tabular}




\begin{tabular}{|c|c|c|c|}
\hline $\mathrm{H}$ & 21.39487300 & 14.07362500 & 24.82318500 \\
\hline $\mathrm{H}$ & 17.96053600 & 14.93968000 & 21.89945700 \\
\hline $\mathrm{H}$ & 17.20467900 & 13.36437900 & 22.13637600 \\
\hline $\mathrm{H}$ & 17.87201500 & 13.81355300 & 20.55156800 \\
\hline $\mathrm{H}$ & 18.52613100 & 12.15779600 & 19.61391900 \\
\hline $\mathrm{H}$ & 17.05466900 & 11.59237000 & 20.40226200 \\
\hline $\mathrm{H}$ & 18.01444300 & 10.49808400 & 19.41296100 \\
\hline $\mathrm{H}$ & 22.56985900 & 7.91254600 & 25.20366100 \\
\hline $\mathrm{H}$ & 20.86629400 & 7.63853300 & 24.76442300 \\
\hline $\mathrm{H}$ & 22.07214800 & 12.95835100 & 27.41086800 \\
\hline $\mathrm{H}$ & 18.90627300 & 11.72002900 & 25.95996900 \\
\hline $\mathrm{H}$ & 18.24050500 & 9.34725800 & 25.87481500 \\
\hline $\mathrm{H}$ & 19.52145400 & 7.63943700 & 27.15802500 \\
\hline $\mathrm{H}$ & 21.51575900 & 8.34290100 & 28.49622400 \\
\hline $\mathrm{H}$ & 22.17982900 & 10.70660600 & 28.55679400 \\
\hline $\mathrm{H}$ & 25.46749500 & 9.15385200 & 32.43322000 \\
\hline $\mathrm{H}$ & 20.50757100 & 25.61931900 & 28.43623300 \\
\hline $\mathrm{H}$ & 20.11437100 & 24.26862900 & 27.35839600 \\
\hline $\mathrm{H}$ & 23.95749500 & 17.28894200 & 23.24640600 \\
\hline $\mathrm{H}$ & 23.05504500 & 15.78687600 & 23.45133600 \\
\hline $\mathrm{H}$ & 22.15171700 & 7.52429500 & 23.51814300 \\
\hline $\mathrm{H}$ & 26.97702100 & 8.25399700 & 32.19670900 \\
\hline $\mathrm{C}$ & 19.56761300 & 13.92071400 & 23.52232200 \\
\hline $\mathrm{H}$ & 18.69397800 & 13.65148400 & 24.13997400 \\
\hline $\mathrm{H}$ & 19.53990200 & 15.01366100 & 23.51411900 \\
\hline $\mathrm{C}$ & 18.29101200 & 8.47891500 & 20.79226100 \\
\hline $\mathrm{H}$ & 17.21942800 & 8.70174200 & 20.76237500 \\
\hline $\mathrm{H}$ & 18.63683600 & 8.42110600 & 19.75299300 \\
\hline $\mathrm{H}$ & 18.41305600 & 7.48606500 & 21.23280500 \\
\hline $\mathrm{N}$ & 24.54306600 & 11.99291700 & 25.56411800 \\
\hline $\mathrm{H}$ & 25.27133400 & 12.58714600 & 25.93820800 \\
\hline $\mathrm{C}$ & 18.72526900 & 14.00067600 & 27.48865700 \\
\hline $\mathrm{H}$ & 18.26725300 & 13.04626100 & 27.75935800 \\
\hline $\mathrm{H}$ & 18.44339900 & 14.73730100 & 28.25292100 \\
\hline $\mathrm{H}$ & 18.27149700 & 14.34477600 & 26.54880300 \\
\hline $\mathrm{C}$ & 13.12103300 & 15.38296200 & 22.77302000 \\
\hline $\mathrm{C}$ & 14.43708400 & 15.05629300 & 23.49147200 \\
\hline $\mathrm{C}$ & 15.53437000 & 16.09126400 & 23.24170700 \\
\hline $\mathrm{C}$ & 16.83254900 & 15.87174800 & 24.04874200 \\
\hline $\mathrm{O}$ & 17.83764100 & 16.55666900 & 23.65940400 \\
\hline $\mathrm{H}$ & 14.27771100 & 14.97639200 & 24.57167900 \\
\hline $\mathrm{H}$ & 14.79807100 & 14.06821600 & 23.17659600 \\
\hline $\mathrm{H}$ & 15.79596400 & 16.14335500 & 22.17733400 \\
\hline $\mathrm{H}$ & 15.17107600 & 17.09675100 & 23.49997000 \\
\hline
\end{tabular}




$\begin{array}{llll}\mathrm{H} & 13.26407500 & 15.44250100 & 21.68690800 \\ \mathrm{H} & 12.72332400 & 16.35084700 & 23.10168500 \\ \mathrm{H} & 12.35378900 & 14.62429500 & 22.96682500 \\ \mathrm{O} & 16.81186900 & 15.07447500 & 25.01578900 \\ \mathrm{H} & 20.73787000 & 14.87210000 & 27.42020500\end{array}$

TS3b

\begin{tabular}{|c|c|c|c|}
\hline $\mathrm{C}$ & 23.14202700 & 16.68499500 & 22.83200600 \\
\hline $\mathrm{C}$ & 21.65446400 & 17.04698600 & 22.81683900 \\
\hline $\mathrm{C}$ & 21.19836300 & 17.64053800 & 24.15151400 \\
\hline $\mathrm{C}$ & 19.75025400 & 18.14768900 & 24.13262900 \\
\hline $\mathrm{N}$ & 19.41578200 & 18.86067500 & 25.36689300 \\
\hline $\mathrm{C}$ & 19.15416200 & 18.26281900 & 26.52804800 \\
\hline $\mathrm{N}$ & 18.89528100 & 16.93099900 & 26.59811800 \\
\hline $\mathrm{N}$ & 19.14510800 & 19.01605400 & 27.64176200 \\
\hline $\mathrm{C}$ & 26.03293300 & 8.54205700 & 31.72090600 \\
\hline $\mathrm{C}$ & 26.51047400 & 8.81782500 & 30.29156200 \\
\hline $\mathrm{C}$ & 25.54371200 & 9.69757400 & 29.47580600 \\
\hline $\mathrm{C}$ & 24.24616300 & 8.97559200 & 29.11907300 \\
\hline $\mathrm{O}$ & 23.50307700 & 8.49471900 & 29.98099300 \\
\hline $\mathrm{N}$ & 23.98838100 & 8.88764300 & 27.78974800 \\
\hline $\mathrm{C}$ & 20.93702000 & 24.79706000 & 27.85204100 \\
\hline $\mathrm{C}$ & 22.07060000 & 23.76656800 & 27.78375400 \\
\hline $\mathrm{C}$ & 22.00966600 & 22.87058500 & 26.54120000 \\
\hline $\mathrm{C}$ & 20.95660200 & 21.73828400 & 26.56265200 \\
\hline $\mathrm{O}$ & 20.50416000 & 21.36999800 & 27.68549900 \\
\hline $\mathrm{O}$ & 20.67896100 & 21.23625400 & 25.43845800 \\
\hline $\mathrm{C}$ & 19.90536500 & 8.34665900 & 22.33885100 \\
\hline $\mathrm{C}$ & 19.03140300 & 8.46997400 & 21.26127300 \\
\hline $\mathrm{C}$ & 18.50537600 & 9.73174300 & 20.90764100 \\
\hline $\mathrm{C}$ & 21.88927100 & 10.50947800 & 24.56509700 \\
\hline $\mathrm{C}$ & 18.78438000 & 10.85089200 & 21.72308300 \\
\hline $\mathrm{C}$ & 23.66346400 & 11.44174000 & 25.73308600 \\
\hline $\mathrm{C}$ & 19.20980500 & 13.14065200 & 20.75503700 \\
\hline $\mathrm{C}$ & 18.20561300 & 12.27149700 & 21.54684700 \\
\hline $\mathrm{C}$ & 19.21670600 & 12.83956700 & 23.82910800 \\
\hline $\mathrm{O}$ & 24.65016700 & 11.37334800 & 26.47905800 \\
\hline $\mathrm{N}$ & 22.97579600 & 10.34167100 & 25.30512200 \\
\hline $\mathrm{C}$ & 22.09263600 & 12.95206600 & 24.52344200 \\
\hline $\mathrm{O}$ & 21.81000100 & 14.11659700 & 24.20611600 \\
\hline $\mathrm{C}$ & 21.34538000 & 11.76027100 & 24.20882200 \\
\hline $\mathrm{N}$ & 20.10402100 & 11.82637100 & 23.55054500 \\
\hline $\mathrm{C}$ & 16.81387300 & 12.36398400 & 20.88103700 \\
\hline
\end{tabular}




\begin{tabular}{|c|c|c|c|}
\hline $\mathrm{C}$ & 19.70186900 & 10.69936100 & 22.78791400 \\
\hline $\mathrm{C}$ & 17.71288100 & 9.83264200 & 19.61828000 \\
\hline $\mathrm{C}$ & 20.29543300 & 9.45731100 & 23.08921100 \\
\hline $\mathrm{N}$ & 21.24544800 & 9.35212700 & 24.11634400 \\
\hline $\mathrm{C}$ & 21.82273500 & 8.04299200 & 24.42629700 \\
\hline $\mathrm{C}$ & 18.63915800 & 12.51654600 & 25.69752100 \\
\hline $\mathrm{C}$ & 19.68128400 & 13.06529300 & 26.44830800 \\
\hline $\mathrm{C}$ & 20.60064900 & 12.53623500 & 27.42636900 \\
\hline $\mathrm{C}$ & 20.59990100 & 11.21408900 & 27.93684200 \\
\hline $\mathrm{C}$ & 21.50116900 & 10.83013700 & 28.92129100 \\
\hline $\mathrm{C}$ & 22.43513300 & 11.74303300 & 29.42307200 \\
\hline $\mathrm{C}$ & 22.46311300 & 13.04888000 & 28.92969400 \\
\hline $\mathrm{C}$ & 21.55959400 & 13.43986200 & 27.94960500 \\
\hline $\mathrm{H}$ & 21.06795800 & 16.14610900 & 22.60193000 \\
\hline $\mathrm{H}$ & 21.45348900 & 17.76596500 & 22.00880100 \\
\hline $\mathrm{H}$ & 21.30298000 & 16.87981200 & 24.93461100 \\
\hline $\mathrm{H}$ & 21.84437800 & 18.48525500 & 24.42672000 \\
\hline $\mathrm{H}$ & 19.03891300 & 17.33734000 & 23.95143200 \\
\hline $\mathrm{H}$ & 19.62540300 & 18.87508100 & 23.32295900 \\
\hline $\mathrm{H}$ & 19.81707200 & 19.83444800 & 25.43238100 \\
\hline $\mathrm{H}$ & 18.48899500 & 16.60177700 & 27.46189600 \\
\hline $\mathrm{H}$ & 18.53184700 & 16.41521200 & 25.76612100 \\
\hline $\mathrm{H}$ & 19.10796200 & 18.52379300 & 28.51853100 \\
\hline $\mathrm{H}$ & 19.63572500 & 19.95674700 & 27.64384800 \\
\hline $\mathrm{H}$ & 23.46533200 & 16.27415300 & 21.86816100 \\
\hline $\mathrm{H}$ & 27.48798700 & 9.31658800 & 30.31992000 \\
\hline $\mathrm{H}$ & 26.66631400 & 7.86901600 & 29.76069700 \\
\hline $\mathrm{H}$ & 25.26777900 & 10.57545700 & 30.07277100 \\
\hline $\mathrm{H}$ & 26.02728800 & 10.07382100 & 28.57002900 \\
\hline $\mathrm{H}$ & 24.37845800 & 9.60156600 & 27.17044900 \\
\hline $\mathrm{H}$ & 23.04715600 & 8.60535300 & 27.55465900 \\
\hline $\mathrm{H}$ & 25.05753800 & 8.05418900 & 31.71251300 \\
\hline $\mathrm{H}$ & 22.02612800 & 23.11948200 & 28.66636600 \\
\hline $\mathrm{H}$ & 23.03631800 & 24.29109200 & 27.80987800 \\
\hline $\mathrm{H}$ & 22.97478400 & 22.36374400 & 26.40258900 \\
\hline $\mathrm{H}$ & 21.84969200 & 23.45973700 & 25.63038700 \\
\hline $\mathrm{H}$ & 20.95724900 & 25.46826200 & 26.98441700 \\
\hline $\mathrm{H}$ & 20.30711700 & 7.36920500 & 22.57150600 \\
\hline $\mathrm{H}$ & 20.17812800 & 13.19901200 & 21.25933800 \\
\hline $\mathrm{H}$ & 18.82356200 & 14.16049900 & 20.65131900 \\
\hline $\mathrm{H}$ & 19.37038800 & 12.73000900 & 19.75248600 \\
\hline $\mathrm{H}$ & 19.68197700 & 13.80052200 & 23.99394400 \\
\hline $\mathrm{H}$ & 16.39642700 & 13.35263800 & 21.09469700 \\
\hline $\mathrm{H}$ & 16.12361000 & 11.61906900 & 21.28833600 \\
\hline
\end{tabular}




\begin{tabular}{|c|c|c|c|}
\hline $\mathrm{H}$ & 16.84109000 & 12.25973900 & 19.79684200 \\
\hline $\mathrm{H}$ & 17.96009700 & 10.74243400 & 19.06983800 \\
\hline $\mathrm{H}$ & 16.62892400 & 9.82684200 & 19.77603500 \\
\hline $\mathrm{H}$ & 17.94440500 & 8.99802100 & 18.95535100 \\
\hline $\mathrm{H}$ & 22.54319900 & 8.17915000 & 25.22775900 \\
\hline $\mathrm{H}$ & 21.03263200 & 7.35326000 & 24.73723200 \\
\hline $\mathrm{H}$ & 19.80441200 & 14.13470900 & 26.27816100 \\
\hline $\mathrm{H}$ & 19.88855900 & 10.49006000 & 27.56584300 \\
\hline $\mathrm{H}$ & 21.50606700 & 9.81416100 & 29.30309500 \\
\hline $\mathrm{H}$ & 23.12996100 & 11.42505400 & 30.19313100 \\
\hline $\mathrm{H}$ & 23.19263400 & 13.75978700 & 29.30664100 \\
\hline $\mathrm{H}$ & 21.57732100 & 14.45576800 & 27.56410800 \\
\hline $\mathrm{H}$ & 25.92827700 & 9.48166300 & 32.27709600 \\
\hline $\mathrm{H}$ & 21.00207000 & 25.41313900 & 28.75641000 \\
\hline $\mathrm{H}$ & 19.96867900 & 24.28530700 & 27.85926100 \\
\hline $\mathrm{H}$ & 23.76010400 & 17.56584600 & 23.04514100 \\
\hline $\mathrm{H}$ & 23.33207300 & 15.92675700 & 23.59539300 \\
\hline $\mathrm{H}$ & 22.34362100 & 7.62479500 & 23.55633600 \\
\hline $\mathrm{H}$ & 26.74934500 & 7.91224400 & 32.26189000 \\
\hline $\mathrm{C}$ & 17.98549600 & 12.85819700 & 22.96194400 \\
\hline $\mathrm{H}$ & 17.16379100 & 12.31579300 & 23.44111700 \\
\hline $\mathrm{H}$ & 17.67561600 & 13.89985400 & 22.88596600 \\
\hline $\mathrm{C}$ & 18.69121300 & 7.21888600 & 20.47869400 \\
\hline $\mathrm{H}$ & 17.61075200 & 7.10060300 & 20.34321900 \\
\hline $\mathrm{H}$ & 19.14160900 & 7.22277800 & 19.47813000 \\
\hline $\mathrm{H}$ & 19.05904000 & 6.32852400 & 20.99581200 \\
\hline $\mathrm{N}$ & 23.23640800 & 12.68838600 & 25.27375200 \\
\hline $\mathrm{H}$ & 23.75264200 & 13.49576300 & 25.59659200 \\
\hline $\mathrm{C}$ & 18.20890200 & 11.07022000 & 25.77621000 \\
\hline $\mathrm{H}$ & 19.04405700 & 10.38104700 & 25.62091800 \\
\hline $\mathrm{H}$ & 17.76407400 & 10.85271100 & 26.75387700 \\
\hline $\mathrm{H}$ & 17.45038800 & 10.85116100 & 25.01972000 \\
\hline $\mathrm{C}$ & 13.12105100 & 15.38291300 & 22.77301400 \\
\hline $\mathrm{C}$ & 14.27310700 & 14.79471300 & 23.59486700 \\
\hline $\mathrm{C}$ & 15.46128900 & 15.74642700 & 23.76973600 \\
\hline $\mathrm{C}$ & 16.56021500 & 15.18539400 & 24.69948000 \\
\hline $\mathrm{O}$ & 17.75137500 & 15.60326200 & 24.49175200 \\
\hline $\mathrm{H}$ & 13.92738900 & 14.50223100 & 24.59134000 \\
\hline $\mathrm{H}$ & 14.62518100 & 13.86769000 & 23.12252100 \\
\hline $\mathrm{H}$ & 15.91850900 & 16.00410700 & 22.80654700 \\
\hline $\mathrm{H}$ & 15.11682500 & 16.69705700 & 24.20335700 \\
\hline $\mathrm{H}$ & 13.44908000 & 15.65746200 & 21.76244200 \\
\hline $\mathrm{H}$ & 12.72530400 & 16.29166100 & 23.24322600 \\
\hline $\mathrm{H}$ & 12.29248100 & 14.67225200 & 22.67183200 \\
\hline
\end{tabular}


$\mathrm{O}$

$\mathrm{H}$

Int2c

C

C

C

C

$\mathrm{N}$

C

$\mathrm{N}$

$\mathrm{N}$

C

C

C

C

O

$\mathrm{N}$

C

C

C

C

O

O

C

C

C

C

C

C

C

C

C

O

$\mathrm{N}$

C

$\mathrm{O}$

C

$\mathrm{N}$

C

C

C

C

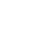

C

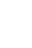

C

C

C

(

C

(n)

.

C

C

O

C

C

C

C

C

C

C

O

$\mathrm{N}$

(1)
16.21900200

14.34775000

13.24329800
25.56485000

25.56985400
C

C

23.26869800

22.33957300

21.35851700

18.93967500

20.94715900

$\begin{array}{ll}C & 19.65506500\end{array}$

21.17499800

23.14202000

16.68501300

17.03677200

17.45348500

17.80702300

18.49483000

17.90712800

16.56841800

18.66298800

8.54203500

9.43813500

10.78238900

10.66206400

11.10998700

10.02187400

24.79701200

23.92868000

22.85176400

21.59666800

21.24780900

20.97503700

8.87361200

9.24772500

10.60644400

10.40048700

11.58459700

10.82399000

13.89326800

13.09849100

13.28317000

10.52446800

9.93635200

12.69490800

13.92422200

11.74024600

12.13878300

13.51757900

11.18403700

10.96493700

9.81984700

22.83201600

23.21819800

24.68599700

25.09469400

26.38128100

27.56143800

27.71903400

28.62463800

31.72095200

30.47880800

30.62390000

30.81634800

31.81385800

29.80538000

27.85200500

27.82506000

26.73398100

27.04618400

28.25601200

26.03972800

21.87655700

20.63433000

20.33362600

24.81299700

21.32294200

26.47001600

20.91873500

21.24487400

23.78808900

27.45795400

25.78853400

25.01927300

24.78058200

24.42869800

23.47822700

20.25925800

22.54270000

18.92317200

22.83705900 


\begin{tabular}{|c|c|c|c|}
\hline $\mathrm{N}$ & 21.71526300 & 9.45426300 & 24.08612200 \\
\hline $\mathrm{C}$ & 21.82273100 & 8.04300000 & 24.42626900 \\
\hline $\mathrm{C}$ & 19.85245500 & 13.06086700 & 25.16198700 \\
\hline $\mathrm{C}$ & 20.48337500 & 13.58171800 & 26.24687000 \\
\hline $\mathrm{C}$ & 20.41797400 & 13.18618600 & 27.65484800 \\
\hline $\mathrm{C}$ & 19.39868000 & 12.39988600 & 28.22608600 \\
\hline $\mathrm{C}$ & 19.49939100 & 11.96612800 & 29.54786800 \\
\hline $\mathrm{C}$ & 20.61142400 & 12.29361700 & 30.32881000 \\
\hline $\mathrm{C}$ & 21.61899000 & 13.09292400 & 29.78216500 \\
\hline $\mathrm{C}$ & 21.51541300 & 13.54580700 & 28.46991800 \\
\hline $\mathrm{H}$ & 21.05290600 & 16.17316200 & 23.02850800 \\
\hline $\mathrm{H}$ & 21.32579700 & 17.85163000 & 22.58368300 \\
\hline $\mathrm{H}$ & 21.97594400 & 16.65218500 & 25.32660200 \\
\hline $\mathrm{H}$ & 22.20607400 & 18.33962500 & 24.86799900 \\
\hline $\mathrm{H}$ & 19.49369500 & 16.92677800 & 25.08242000 \\
\hline $\mathrm{H}$ & 19.72370900 & 18.50445000 & 24.36612300 \\
\hline $\mathrm{H}$ & 20.33164500 & 19.53837900 & 26.32540700 \\
\hline $\mathrm{H}$ & 20.46597800 & 16.09318300 & 28.50923500 \\
\hline $\mathrm{H}$ & 19.95424200 & 15.98154100 & 26.90444200 \\
\hline $\mathrm{H}$ & 20.49689800 & 18.25555800 & 29.54432800 \\
\hline $\mathrm{H}$ & 20.76124200 & 19.69575100 & 28.50704900 \\
\hline $\mathrm{H}$ & 23.21456700 & 16.43400500 & 21.76795200 \\
\hline $\mathrm{H}$ & 27.17871600 & 9.65047800 & 30.25936500 \\
\hline $\mathrm{H}$ & 25.73216500 & 8.91478300 & 29.60067900 \\
\hline $\mathrm{H}$ & 25.76685600 & 11.34686900 & 31.47995700 \\
\hline $\mathrm{H}$ & 25.54888800 & 11.36139200 & 29.70735700 \\
\hline $\mathrm{H}$ & 23.68536900 & 9.97621400 & 28.88900100 \\
\hline $\mathrm{H}$ & 22.21865300 & 10.11159000 & 29.81071200 \\
\hline $\mathrm{H}$ & 24.99043900 & 8.28999800 & 31.94359300 \\
\hline $\mathrm{H}$ & 22.32440400 & 23.42565600 & 28.78994500 \\
\hline $\mathrm{H}$ & 23.07689100 & 24.57534300 & 27.67953800 \\
\hline $\mathrm{H}$ & 23.19370700 & 22.47740100 & 26.56401400 \\
\hline $\mathrm{H}$ & 21.83662700 & 23.25899400 & 25.77455600 \\
\hline $\mathrm{H}$ & 20.79667100 & 25.32124400 & 26.89894900 \\
\hline $\mathrm{H}$ & 20.97234400 & 7.81978600 & 22.07988500 \\
\hline $\mathrm{H}$ & 22.12459200 & 13.69830700 & 21.64923000 \\
\hline $\mathrm{H}$ & 21.13116100 & 14.97028500 & 20.91493200 \\
\hline $\mathrm{H}$ & 21.70904800 & 13.61660100 & 19.92659800 \\
\hline $\mathrm{H}$ & 21.13703700 & 14.15452500 & 23.87917400 \\
\hline $\mathrm{H}$ & 18.64403000 & 14.54921900 & 20.48132800 \\
\hline $\mathrm{H}$ & 18.05166100 & 12.88634700 & 20.36350500 \\
\hline $\mathrm{H}$ & 19.25721200 & 13.49999600 & 19.21717200 \\
\hline $\mathrm{H}$ & 20.19521700 & 11.83787300 & 18.55099300 \\
\hline $\mathrm{H}$ & 18.58370300 & 11.18006600 & 18.83482100 \\
\hline
\end{tabular}




\begin{tabular}{|c|c|c|c|}
\hline $\mathrm{H}$ & 19.87264100 & 10.15001400 & 18.23110200 \\
\hline $\mathrm{H}$ & 22.20300100 & 7.97616000 & 25.44319300 \\
\hline $\mathrm{H}$ & 20.83813600 & 7.56819300 & 24.35530600 \\
\hline $\mathrm{H}$ & 21.32143600 & 14.22674400 & 25.99681900 \\
\hline $\mathrm{H}$ & 18.55730000 & 12.10322500 & 27.61296900 \\
\hline $\mathrm{H}$ & 18.71081200 & 11.34400900 & 29.96395500 \\
\hline $\mathrm{H}$ & 20.73657300 & 11.90164100 & 31.33271500 \\
\hline $\mathrm{H}$ & 22.50073700 & 13.30948900 & 30.37635300 \\
\hline $\mathrm{H}$ & 22.32431800 & 14.12462800 & 28.02967700 \\
\hline $\mathrm{H}$ & 26.44460000 & 9.04695300 & 32.60294700 \\
\hline $\mathrm{H}$ & 20.98025600 & 25.54923800 & 28.64791300 \\
\hline $\mathrm{H}$ & 20.05146700 & 24.17538100 & 28.02458800 \\
\hline $\mathrm{H}$ & 23.81232300 & 17.53154600 & 23.02321000 \\
\hline $\mathrm{H}$ & 23.49027200 & 15.82216900 & 23.40703600 \\
\hline $\mathrm{H}$ & 22.51923700 & 7.51300100 & 23.76086700 \\
\hline $\mathrm{H}$ & 26.58376500 & 7.60573900 & 31.57779400 \\
\hline $\mathrm{C}$ & 19.49784300 & 13.48736000 & 22.64453700 \\
\hline $\mathrm{H}$ & 18.59356600 & 12.89251500 & 22.80040900 \\
\hline $\mathrm{H}$ & 19.18772900 & 14.53895500 & 22.64552400 \\
\hline $\mathrm{C}$ & 19.98799500 & 8.14775000 & 19.63988000 \\
\hline $\mathrm{H}$ & 18.96783400 & 8.22048100 & 19.24494900 \\
\hline $\mathrm{H}$ & 20.66565900 & 8.15851700 & 18.77575900 \\
\hline $\mathrm{H}$ & 20.08613800 & 7.16556100 & 20.11139700 \\
\hline $\mathrm{N}$ & 24.01608600 & 12.14749700 & 26.02586000 \\
\hline $\mathrm{H}$ & 24.60722900 & 12.79475100 & 26.52900100 \\
\hline $\mathrm{C}$ & 13.12105100 & 15.38295700 & 22.77302200 \\
\hline $\mathrm{C}$ & 14.29688700 & 14.49878000 & 23.20607000 \\
\hline $\mathrm{C}$ & 15.41327900 & 15.28457600 & 23.90033700 \\
\hline $\mathrm{C}$ & 16.57188600 & 14.41085700 & 24.33349700 \\
\hline $\mathrm{O}$ & 17.55368200 & 15.12257800 & 24.90967200 \\
\hline $\mathrm{H}$ & 13.94777500 & 13.71030700 & 23.88163000 \\
\hline $\mathrm{H}$ & 14.71867000 & 13.97987000 & 22.33829600 \\
\hline $\mathrm{H}$ & 15.81952000 & 16.06794400 & 23.24764800 \\
\hline $\mathrm{H}$ & 15.04303700 & 15.80983400 & 24.79021500 \\
\hline $\mathrm{H}$ & 13.44385500 & 16.15760300 & 22.06802000 \\
\hline $\mathrm{H}$ & 12.66535400 & 15.88819900 & 23.63239700 \\
\hline $\mathrm{H}$ & 12.34359200 & 14.78841900 & 22.28345500 \\
\hline $\mathrm{O}$ & 16.60942600 & 13.20279200 & 24.16875200 \\
\hline $\mathrm{H}$ & 18.30146400 & 14.53552100 & 25.16969600 \\
\hline $\mathrm{O}$ & 18.92259300 & 12.06745900 & 25.29968000 \\
\hline $\mathrm{H}$ & 18.19574700 & 12.19356800 & 24.66325100 \\
\hline
\end{tabular}

TS $_{\text {inh }}$ 


\begin{tabular}{|c|c|c|c|}
\hline $\mathrm{C}$ & 23.14196400 & 16.68500100 & 22.83207700 \\
\hline $\mathrm{C}$ & 21.69320800 & 17.16164600 & 22.68048100 \\
\hline $\mathrm{C}$ & 21.17297800 & 17.86097400 & 23.93939600 \\
\hline $\mathrm{C}$ & 19.75610900 & 18.42808000 & 23.77930000 \\
\hline $\mathrm{N}$ & 19.35094400 & 19.21100800 & 24.94780000 \\
\hline $\mathrm{C}$ & 18.96611800 & 18.67675000 & 26.10275200 \\
\hline $\mathrm{N}$ & 18.38174700 & 17.44736900 & 26.15877500 \\
\hline $\mathrm{N}$ & 19.11207700 & 19.37692900 & 27.23063900 \\
\hline $\mathrm{C}$ & 26.03286600 & 8.54211300 & 31.72080300 \\
\hline $\mathrm{C}$ & 25.09065700 & 9.53118700 & 31.02426700 \\
\hline $\mathrm{C}$ & 23.61776400 & 9.07223600 & 31.00774900 \\
\hline $\mathrm{C}$ & 23.44520000 & 7.78973500 & 30.19357400 \\
\hline $\mathrm{O}$ & 23.55125400 & 6.67159600 & 30.70408500 \\
\hline $\mathrm{N}$ & 23.21048900 & 7.99667200 & 28.87574000 \\
\hline $\mathrm{C}$ & 20.93698400 & 24.79690200 & 27.85196700 \\
\hline $\mathrm{C}$ & 22.13248800 & 23.94025700 & 27.42169200 \\
\hline $\mathrm{C}$ & 21.90961100 & 23.21352500 & 26.09183500 \\
\hline $\mathrm{C}$ & 20.91820200 & 22.03567400 & 26.12707100 \\
\hline $\mathrm{O}$ & 20.49450300 & 21.64760400 & 27.25520200 \\
\hline $\mathrm{O}$ & 20.63210500 & 21.53001400 & 25.00323500 \\
\hline $\mathrm{C}$ & 22.06499000 & 8.64557700 & 21.63506300 \\
\hline $\mathrm{C}$ & 22.11867500 & 8.91142400 & 20.26181000 \\
\hline $\mathrm{C}$ & 22.07987700 & 10.23854600 & 19.79500300 \\
\hline $\mathrm{C}$ & 22.37460000 & 10.41084200 & 24.84886500 \\
\hline $\mathrm{C}$ & 21.92847000 & 11.29750900 & 20.72536700 \\
\hline $\mathrm{C}$ & 23.15422800 & 10.96729300 & 26.95662600 \\
\hline $\mathrm{C}$ & 23.05511300 & 13.55840700 & 20.52397100 \\
\hline $\mathrm{C}$ & 21.71985000 & 12.79314700 & 20.39478700 \\
\hline $\mathrm{C}$ & 21.12367700 & 13.21915000 & 22.87129700 \\
\hline $\mathrm{O}$ & 23.44783100 & 10.72659100 & 28.14517900 \\
\hline $\mathrm{N}$ & 22.70326700 & 10.02437900 & 26.08729700 \\
\hline $\mathrm{C}$ & 22.98689700 & 12.73458200 & 25.19889500 \\
\hline $\mathrm{O}$ & 23.13830400 & 13.94373800 & 24.92229800 \\
\hline $\mathrm{C}$ & 22.43399400 & 11.72080000 & 24.36735800 \\
\hline $\mathrm{N}$ & 21.96448900 & 12.03178200 & 23.05585500 \\
\hline $\mathrm{C}$ & 21.09375000 & 13.09829100 & 19.01547900 \\
\hline $\mathrm{C}$ & 21.94824300 & 11.00395700 & 22.10574300 \\
\hline $\mathrm{C}$ & 22.29735700 & 10.47725800 & 18.31217500 \\
\hline $\mathrm{C}$ & 21.99709400 & 9.67113200 & 22.57280600 \\
\hline $\mathrm{N}$ & 21.94782100 & 9.41458400 & 23.96017200 \\
\hline $\mathrm{C}$ & 21.82268400 & 8.04308500 & 24.42632900 \\
\hline $\mathrm{C}$ & 19.95090000 & 13.11536800 & 23.82853800 \\
\hline $\mathrm{C}$ & 19.83887300 & 13.91833500 & 24.97341400 \\
\hline $\mathrm{C}$ & 19.20681500 & 13.36585800 & 26.23106700 \\
\hline
\end{tabular}




\begin{tabular}{|c|c|c|c|}
\hline $\mathrm{C}$ & 18.13837600 & 13.98753300 & 26.89116700 \\
\hline $\mathrm{C}$ & 17.65504500 & 13.48652500 & 28.10163200 \\
\hline $\mathrm{C}$ & 18.23081000 & 12.35144000 & 28.67367500 \\
\hline $\mathrm{C}$ & 19.28615200 & 11.71325700 & 28.01926000 \\
\hline $\mathrm{C}$ & 19.76477800 & 12.21409100 & 26.80987300 \\
\hline $\mathrm{H}$ & 21.04224200 & 16.30793500 & 22.44566200 \\
\hline $\mathrm{H}$ & 21.61304700 & 17.85022400 & 21.82713100 \\
\hline $\mathrm{H}$ & 21.18868600 & 17.15926500 & 24.78388400 \\
\hline $\mathrm{H}$ & 21.83758800 & 18.69149600 & 24.20860700 \\
\hline $\mathrm{H}$ & 19.03526100 & 17.63205400 & 23.56503500 \\
\hline $\mathrm{H}$ & 19.72629800 & 19.11442800 & 22.92701500 \\
\hline $\mathrm{H}$ & 19.79186800 & 20.18230300 & 25.00565800 \\
\hline $\mathrm{H}$ & 18.50951600 & 16.90540000 & 26.99949100 \\
\hline $\mathrm{H}$ & 18.23671000 & 16.90367300 & 25.31289200 \\
\hline $\mathrm{H}$ & 18.58751200 & 19.09045200 & 28.04179900 \\
\hline $\mathrm{H}$ & 19.64872900 & 20.30306300 & 27.24128000 \\
\hline $\mathrm{H}$ & 23.50944600 & 16.23177400 & 21.90614600 \\
\hline $\mathrm{H}$ & 25.14468800 & 10.50676300 & 31.52455700 \\
\hline $\mathrm{H}$ & 25.40548600 & 9.70284900 & 29.98846800 \\
\hline $\mathrm{H}$ & 23.27853600 & 8.85705200 & 32.02656400 \\
\hline $\mathrm{H}$ & 23.00469800 & 9.87326500 & 30.58386800 \\
\hline $\mathrm{H}$ & 23.24057400 & 8.93934200 & 28.46778700 \\
\hline $\mathrm{H}$ & 23.20363300 & 7.18794800 & 28.27357400 \\
\hline $\mathrm{H}$ & 25.96694000 & 7.55060800 & 31.26435400 \\
\hline $\mathrm{H}$ & 22.33346000 & 23.19020600 & 28.19402600 \\
\hline $\mathrm{H}$ & 23.02735600 & 24.57300400 & 27.34192400 \\
\hline $\mathrm{H}$ & 22.85522300 & 22.79667900 & 25.72088800 \\
\hline $\mathrm{H}$ & 21.57016700 & 23.90700200 & 25.31200900 \\
\hline $\mathrm{H}$ & 20.71483200 & 25.56934400 & 27.10496700 \\
\hline $\mathrm{H}$ & 22.09176200 & 7.61420600 & 21.96509200 \\
\hline $\mathrm{H}$ & 23.48543500 & 13.44796300 & 21.52218900 \\
\hline $\mathrm{H}$ & 22.90317900 & 14.62676500 & 20.33037700 \\
\hline $\mathrm{H}$ & 23.77998600 & 13.18207400 & 19.79432500 \\
\hline $\mathrm{H}$ & 21.71831700 & 14.08419500 & 23.16917600 \\
\hline $\mathrm{H}$ & 20.74873700 & 14.13806400 & 19.00707800 \\
\hline $\mathrm{H}$ & 20.22849900 & 12.45787300 & 18.81861000 \\
\hline $\mathrm{H}$ & 21.79630300 & 12.99432200 & 18.18955400 \\
\hline $\mathrm{H}$ & 22.94058300 & 11.34199000 & 18.13908100 \\
\hline $\mathrm{H}$ & 21.36828800 & 10.64023800 & 17.75357100 \\
\hline $\mathrm{H}$ & 22.79825200 & 9.62379500 & 17.85255300 \\
\hline $\mathrm{H}$ & 21.69543500 & 8.06580700 & 25.50636900 \\
\hline $\mathrm{H}$ & 20.95439200 & 7.57232100 & 23.95363100 \\
\hline $\mathrm{H}$ & 20.73864400 & 14.50981400 & 25.15336000 \\
\hline $\mathrm{H}$ & 17.65868300 & 14.85153000 & 26.44706200 \\
\hline
\end{tabular}




$\begin{array}{lrrr}\mathrm{H} & 16.82228200 & 13.98264300 & 28.59355500 \\ \mathrm{H} & 17.85939400 & 11.96631000 & 29.61916600 \\ \mathrm{H} & 19.75433800 & 10.83224900 & 28.44835400 \\ \mathrm{H} & 20.58678300 & 11.71596200 & 26.31247800 \\ \mathrm{H} & 25.76443300 & 8.42683300 & 32.77778300 \\ \mathrm{H} & 21.12263100 & 25.29891200 & 28.80851400 \\ \mathrm{H} & 20.05243300 & 24.16303600 & 27.96236600 \\ \mathrm{H} & 23.79960800 & 17.52839600 & 23.07337100 \\ \mathrm{H} & 23.23724800 & 15.93733500 & 23.62525400 \\ \mathrm{H} & 22.71657300 & 7.44462700 & 24.19886800 \\ \mathrm{H} & 27.07338700 & 8.88316500 & 31.67159300 \\ \mathrm{C} & 20.67631000 & 13.32741400 & 21.41596400 \\ \mathrm{H} & 19.74836900 & 12.76656400 & 21.27323500 \\ \mathrm{H} & 20.45811200 & 14.37974700 & 21.20272200 \\ \mathrm{C} & 22.23160700 & 7.73285600 & 19.31622200 \\ \mathrm{H} & 21.48187000 & 7.76703700 & 18.51734200 \\ \mathrm{H} & 23.21450500 & 7.68010000 & 18.82941100 \\ \mathrm{H} & 22.09110800 & 6.79234400 & 19.85664400 \\ \mathrm{~N} & 23.29998100 & 12.27136900 & 26.48380300 \\ \mathrm{H} & 23.65419300 & 12.95870000 & 27.13499100 \\ \mathrm{C} & 13.12126900 & 15.38291300 & 22.77308500 \\ \mathrm{C} & 14.37831600 & 14.59187100 & 23.14933500 \\ \mathrm{C} & 15.56738800 & 15.48797400 & 23.50452900 \\ \mathrm{C} & 16.83359000 & 14.71396400 & 23.84598700 \\ \mathrm{O} & 17.89558400 & 15.45129200 & 24.03020700 \\ \mathrm{H} & 14.17195900 & 13.92848500 & 23.99584600 \\ \mathrm{H} & 14.66934300 & 13.93274200 & 22.32392900 \\ \mathrm{H} & 15.81220300 & 16.17366300 & 22.68373600 \\ \mathrm{H} & 15.33694600 & 16.13312400 & 24.36336800 \\ \mathrm{H} & 13.30096600 & 16.02626300 & 21.90376100 \\ \mathrm{H} & 12.79518200 & 16.02848000 & 23.59712000 \\ \mathrm{H} & 12.29156000 & 14.71265400 & 22.52634100 \\ \mathrm{O} & 16.80507800 & 13.47711300 & 23.93917700 \\ \mathrm{H} & 18.89039200 & 14.74674700 & 24.41266800 \\ & 1462700 & 12.25563900 & 23.48061800 \\ \mathrm{H} & 12.52364400 & 23.89415300\end{array}$

Int $_{\text {keto }}$
C
C
C
C
N

$\begin{array}{lll}23.14201400 & 16.68498800 & 22.83202900 \\ 21.76741100 & 17.14388200 & 23.32720700 \\ 21.81895900 & 17.66207500 & 24.76656400 \\ 20.44820500 & 18.09350100 & 25.30280900 \\ 20.55534900 & 18.80979600 & 26.57246800\end{array}$




\begin{tabular}{|c|c|c|c|}
\hline $\mathrm{C}$ & 20.79800200 & 18.24259200 & 27.74732000 \\
\hline $\mathrm{N}$ & 20.52280900 & 16.92616600 & 27.97176300 \\
\hline $\mathrm{N}$ & 21.27553600 & 18.98933700 & 28.74811000 \\
\hline $\mathrm{C}$ & 26.03295400 & 8.54204200 & 31.72094200 \\
\hline $\mathrm{C}$ & 26.13061400 & 9.47348600 & 30.50679000 \\
\hline $\mathrm{C}$ & 25.28799900 & 10.75521400 & 30.64424700 \\
\hline $\mathrm{C}$ & 23.78894300 & 10.50768100 & 30.81017200 \\
\hline $\mathrm{O}$ & 23.16381800 & 10.90803100 & 31.79634700 \\
\hline $\mathrm{N}$ & 23.21583100 & 9.80725800 & 29.79476700 \\
\hline $\mathrm{C}$ & 20.93698700 & 24.79698800 & 27.85198900 \\
\hline $\mathrm{C}$ & 22.38958100 & 24.31746600 & 27.76988400 \\
\hline $\mathrm{C}$ & 22.58760700 & 23.22521700 & 26.71448100 \\
\hline $\mathrm{C}$ & 21.89962200 & 21.89211600 & 27.05503200 \\
\hline $\mathrm{O}$ & 21.86903500 & 21.55172600 & 28.27364300 \\
\hline $\mathrm{O}$ & 21.44962700 & 21.22486900 & 26.07833400 \\
\hline $\mathrm{C}$ & 21.17628600 & 8.82991100 & 21.74881700 \\
\hline $\mathrm{C}$ & 20.77143000 & 9.18513900 & 20.45585700 \\
\hline $\mathrm{C}$ & 20.56727900 & 10.53744300 & 20.12826600 \\
\hline $\mathrm{C}$ & 22.53563600 & 10.37465500 & 24.79448300 \\
\hline $\mathrm{C}$ & 20.72949600 & 11.52958200 & 21.12835000 \\
\hline $\mathrm{C}$ & 24.02127700 & 10.77143900 & 26.51553800 \\
\hline $\mathrm{C}$ & 21.67225600 & 13.83968600 & 20.67439900 \\
\hline $\mathrm{C}$ & 20.39533600 & 13.03188100 & 20.99081000 \\
\hline $\mathrm{C}$ & 20.76839100 & 13.31626300 & 23.51956100 \\
\hline $\mathrm{O}$ & 24.70630600 & 10.45672700 & 27.51373000 \\
\hline $\mathrm{N}$ & 23.26692700 & 9.89714900 & 25.80613800 \\
\hline $\mathrm{C}$ & 23.27139100 & 12.66670400 & 25.07075400 \\
\hline $\mathrm{O}$ & 23.32315700 & 13.90739400 & 24.88437300 \\
\hline $\mathrm{C}$ & 22.44678800 & 11.71740900 & 24.41055900 \\
\hline $\mathrm{N}$ & 21.56029200 & 12.10451200 & 23.35957100 \\
\hline $\mathrm{C}$ & 19.29704500 & 13.38408700 & 19.96218500 \\
\hline $\mathrm{C}$ & 21.21971300 & 11.15079700 & 22.39785100 \\
\hline $\mathrm{C}$ & 20.26776900 & 10.88127000 & 18.68046600 \\
\hline $\mathrm{C}$ & 21.41654600 & 9.78960600 & 22.72612500 \\
\hline $\mathrm{N}$ & 21.81309700 & 9.44282700 & 24.03673700 \\
\hline $\mathrm{C}$ & 21.82272200 & 8.04304200 & 24.42626000 \\
\hline $\mathrm{C}$ & 19.98578200 & 13.24623100 & 24.84666900 \\
\hline $\mathrm{C}$ & 20.33335000 & 14.24529000 & 25.93823500 \\
\hline $\mathrm{C}$ & 20.57760400 & 13.63803800 & 27.30424400 \\
\hline $\mathrm{C}$ & 19.56261700 & 12.96449500 & 27.99528100 \\
\hline $\mathrm{C}$ & 19.81733200 & 12.39877800 & 29.24562900 \\
\hline $\mathrm{C}$ & 21.08415400 & 12.50005400 & 29.82627800 \\
\hline $\mathrm{C}$ & 22.09667200 & 13.17662700 & 29.14205800 \\
\hline $\mathrm{C}$ & 21.84656700 & 13.74461200 & 27.89135200 \\
\hline
\end{tabular}




\begin{tabular}{|c|c|c|c|}
\hline $\mathrm{H}$ & 21.05324800 & 16.31236200 & 23.26040400 \\
\hline $\mathrm{H}$ & 21.37279400 & 17.93695800 & 22.67655400 \\
\hline $\mathrm{H}$ & 22.25061500 & 16.89375800 & 25.42139300 \\
\hline $\mathrm{H}$ & 22.48486300 & 18.53224300 & 24.82257900 \\
\hline $\mathrm{H}$ & 19.75666400 & 17.24494400 & 25.37320400 \\
\hline $\mathrm{H}$ & 19.98454400 & 18.79551500 & 24.60303500 \\
\hline $\mathrm{H}$ & 20.86160200 & 19.83274500 & 26.45898400 \\
\hline $\mathrm{H}$ & 21.04206700 & 16.42987700 & 28.68061100 \\
\hline $\mathrm{H}$ & 20.26639300 & 16.35140400 & 27.18636600 \\
\hline $\mathrm{H}$ & 21.21532600 & 18.62925600 & 29.68713900 \\
\hline $\mathrm{H}$ & 21.49076800 & 20.02092700 & 28.58647900 \\
\hline $\mathrm{H}$ & 23.10316100 & 16.37712200 & 21.78185500 \\
\hline $\mathrm{H}$ & 27.17735900 & 9.76602600 & 30.35187800 \\
\hline $\mathrm{H}$ & 25.82458500 & 8.94771300 & 29.59645300 \\
\hline $\mathrm{H}$ & 25.60592100 & 11.34131400 & 31.51187600 \\
\hline $\mathrm{H}$ & 25.42265200 & 11.35160200 & 29.73436700 \\
\hline $\mathrm{H}$ & 23.68805400 & 9.80663800 & 28.88240200 \\
\hline $\mathrm{H}$ & 22.20576700 & 9.79793600 & 29.79106200 \\
\hline $\mathrm{H}$ & 25.00124700 & 8.20719700 & 31.87266500 \\
\hline $\mathrm{H}$ & 22.69452900 & 23.91117400 & 28.73992100 \\
\hline $\mathrm{H}$ & 23.04800400 & 25.16833500 & 27.54752000 \\
\hline $\mathrm{H}$ & 23.65829000 & 23.00177000 & 26.60879000 \\
\hline $\mathrm{H}$ & 22.23524700 & 23.55189800 & 25.73010600 \\
\hline $\mathrm{H}$ & 20.59712200 & 25.19906900 & 26.88981900 \\
\hline $\mathrm{H}$ & 21.31380900 & 7.78014200 & 21.97853200 \\
\hline $\mathrm{H}$ & 22.45053400 & 13.65977900 & 21.41978100 \\
\hline $\mathrm{H}$ & 21.45383600 & 14.91430400 & 20.65831700 \\
\hline $\mathrm{H}$ & 22.06865200 & 13.55747100 & 19.69327300 \\
\hline $\mathrm{H}$ & 21.45576400 & 14.16140300 & 23.56904300 \\
\hline $\mathrm{H}$ & 18.95453600 & 14.40824800 & 20.14799400 \\
\hline $\mathrm{H}$ & 18.43276000 & 12.71958300 & 20.06070500 \\
\hline $\mathrm{H}$ & 19.64250000 & 13.35085100 & 18.92965500 \\
\hline $\mathrm{H}$ & 20.79074600 & 11.78733700 & 18.36836600 \\
\hline $\mathrm{H}$ & 19.20047600 & 11.03071700 & 18.47814600 \\
\hline $\mathrm{H}$ & 20.60760900 & 10.08496900 & 18.01581600 \\
\hline $\mathrm{H}$ & 22.05093900 & 7.99060900 & 25.48840700 \\
\hline $\mathrm{H}$ & 20.84130000 & 7.60080400 & 24.22489900 \\
\hline $\mathrm{H}$ & 21.20513100 & 14.81920400 & 25.62162600 \\
\hline $\mathrm{H}$ & 18.58569800 & 12.86564300 & 27.53407100 \\
\hline $\mathrm{H}$ & 19.02289200 & 11.87098200 & 29.76701600 \\
\hline $\mathrm{H}$ & 21.30706400 & 12.03917100 & 30.78442400 \\
\hline $\mathrm{H}$ & 23.08894500 & 13.23516900 & 29.57952400 \\
\hline $\mathrm{H}$ & 22.63877400 & 14.23661700 & 27.33398600 \\
\hline $\mathrm{H}$ & 26.35170900 & 9.05244900 & 32.63753300 \\
\hline
\end{tabular}




$\begin{array}{lrrr}\mathrm{H} & 20.81098300 & 25.58090400 & 28.60765800 \\ \mathrm{H} & 20.28045500 & 23.96258400 & 28.11898100 \\ \mathrm{H} & 23.87517700 & 17.49591900 & 22.91635600 \\ \mathrm{H} & 23.49601500 & 15.83151700 & 23.41912700 \\ \mathrm{H} & 22.58755300 & 7.46601900 & 23.88621700 \\ \mathrm{H} & 26.66040000 & 7.65302800 & 31.59228100 \\ \mathrm{C} & 19.79740900 & 13.46875800 & 22.35529500 \\ \mathrm{H} & 18.90737700 & 12.86048600 & 22.54543700 \\ \mathrm{H} & 19.47635200 & 14.51451400 & 22.31269500 \\ \mathrm{C} & 20.57502200 & 8.07573900 & 19.44294000 \\ \mathrm{H} & 19.59904300 & 8.13172700 & 18.94645000 \\ \mathrm{H} & 21.33604700 & 8.09158600 & 18.65113700 \\ \mathrm{H} & 20.63876700 & 7.09743100 & 19.92839000 \\ \mathrm{~N} & 24.04100500 & 12.09966300 & 26.09399200 \\ \mathrm{H} & 24.60862400 & 12.73839000 & 26.63308800 \\ \mathrm{C} & 13.12109300 & 15.38295700 & 22.77304300 \\ \mathrm{C} & 14.25544200 & 14.78079500 & 23.60856300 \\ \mathrm{C} & 15.64125900 & 15.23562100 & 23.12425300 \\ \mathrm{C} & 16.80266700 & 14.64495800 & 23.89958300 \\ \mathrm{O} & 17.75806300 & 15.29503600 & 24.29028000 \\ \mathrm{H} & 14.13113900 & 15.06473700 & 24.66106100 \\ \mathrm{H} & 14.21099200 & 13.68785900 & 23.57576000 \\ \mathrm{H} & 15.77598600 & 14.93544100 & 22.07567700 \\ \mathrm{H} & 15.74344500 & 16.32325300 & 23.16765800 \\ \mathrm{H} & 13.20642600 & 15.08614700 & 21.72126700 \\ \mathrm{H} & 13.13843200 & 16.47823700 & 22.80984500 \\ \mathrm{H} & 12.14276000 & 15.05146600 & 23.13573900 \\ \mathrm{O} & 16.67142100 & 13.32424500 & 24.07456000 \\ \mathrm{H} & 19.44554000 & 14.89417500 & 25.94442000 \\ \mathrm{O} & 19.08021500 & 12.43389900 & 24.99507200 \\ \mathrm{H} & 17.48395700 & 12.96674000 & 24.50776500 \\ & & & \\ & & \end{array}$

\section{TS3c}

$\mathrm{C}$
$\mathrm{C}$
$\mathrm{C}$
$\mathrm{N}$
$\mathrm{C}$
$\mathrm{N}$
$\mathrm{N}$
$\mathrm{C}$
$\mathrm{C}$

$\begin{array}{rrr}-2.53064600 & -0.29153300 & -4.02659500 \\ -3.09900400 & -1.09790700 & -2.85600400 \\ -3.86307500 & -0.21410300 & -1.86681500 \\ -4.62099100 & -1.01930500 & -0.80271600 \\ -5.47641600 & -0.16338200 & 0.01936100 \\ -5.04746000 & 0.57109700 & 1.03621500 \\ -3.79704500 & 0.42703300 & 1.54482800 \\ -5.88685900 & 1.47339900 & 1.58318900 \\ 5.64998000 & 8.17271100 & -0.13925000 \\ 4.54386700 & 7.25138400 & -0.66502200\end{array}$




\begin{tabular}{|c|c|c|c|}
\hline $\mathrm{C}$ & 4.03064600 & 6.25390700 & 0.39237400 \\
\hline $\mathrm{C}$ & 5.13215700 & 5.30206400 & 0.85441300 \\
\hline $\mathrm{O}$ & 5.77014200 & 5.49627000 & 1.89373400 \\
\hline $\mathrm{N}$ & 5.38120300 & 4.27583600 & 0.00221500 \\
\hline $\mathrm{C}$ & -11.05778900 & 3.09464800 & -0.60841100 \\
\hline $\mathrm{C}$ & -9.93484100 & 3.56256800 & -1.53998000 \\
\hline $\mathrm{C}$ & -9.06066000 & 2.41347600 & -2.05067500 \\
\hline $\mathrm{C}$ & -8.11361300 & 1.77888200 & -1.01269100 \\
\hline $\mathrm{O}$ & -7.92652100 & 2.39478300 & 0.07643600 \\
\hline $\mathrm{O}$ & -7.56758400 & 0.69470000 & -1.36825200 \\
\hline $\mathrm{C}$ & 4.98884600 & -3.00153700 & -1.47962200 \\
\hline $\mathrm{C}$ & 4.67607900 & -4.32306000 & -1.80654900 \\
\hline $\mathrm{C}$ & 3.33012000 & -4.74335700 & -1.88141700 \\
\hline $\mathrm{C}$ & 3.30513900 & 0.25407100 & -1.12639300 \\
\hline $\mathrm{C}$ & 2.30102700 & -3.82769700 & -1.55782000 \\
\hline $\mathrm{C}$ & 2.76137600 & 2.48555000 & -1.40892900 \\
\hline $\mathrm{C}$ & 0.04182200 & -3.60525900 & -2.69475400 \\
\hline $\mathrm{C}$ & 0.78435900 & -4.11702900 & -1.43788200 \\
\hline $\mathrm{C}$ & 0.57423900 & -1.87649000 & -0.12632900 \\
\hline $\mathrm{O}$ & 3.02571300 & 3.69937000 & -1.47657600 \\
\hline $\mathrm{N}$ & 3.69870400 & 1.52346700 & -1.19058700 \\
\hline $\mathrm{C}$ & 0.95140500 & 0.77518700 & -1.56161700 \\
\hline $\mathrm{O}$ & -0.25369800 & 0.54438800 & -1.75172600 \\
\hline $\mathrm{C}$ & 1.96608200 & -0.18559100 & -1.22203000 \\
\hline $\mathrm{N}$ & 1.62880100 & -1.54213500 & -1.10095000 \\
\hline $\mathrm{C}$ & 0.38932200 & -5.59088300 & -1.20046100 \\
\hline $\mathrm{C}$ & 2.64824800 & -2.48568800 & -1.29212700 \\
\hline $\mathrm{C}$ & 3.05328500 & -6.14772600 & -2.38710200 \\
\hline $\mathrm{C}$ & 3.99054000 & -2.05967200 & -1.24080900 \\
\hline $\mathrm{N}$ & 4.28799800 & -0.71358400 & -0.94801300 \\
\hline $\mathrm{C}$ & 5.67377800 & -0.30832800 & -0.73136800 \\
\hline $\mathrm{C}$ & 1.08762400 & -1.36526600 & 1.25298000 \\
\hline $\mathrm{C}$ & 1.18877200 & 0.03396800 & 1.31363000 \\
\hline $\mathrm{C}$ & 2.20000500 & 0.89317700 & 1.88275200 \\
\hline $\mathrm{C}$ & 3.45658600 & 0.43561900 & 2.34740900 \\
\hline $\mathrm{C}$ & 4.42563000 & 1.34318300 & 2.76301700 \\
\hline $\mathrm{C}$ & 4.18422400 & 2.71971500 & 2.73207600 \\
\hline $\mathrm{C}$ & 2.94422000 & 3.18556700 & 2.28493200 \\
\hline $\mathrm{C}$ & 1.96865500 & 2.28953400 & 1.86880000 \\
\hline $\mathrm{H}$ & -2.27783200 & -1.60116200 & -2.33195900 \\
\hline $\mathrm{H}$ & -3.76728600 & -1.88537700 & -3.23566600 \\
\hline $\mathrm{H}$ & -3.15926400 & 0.47157100 & -1.38015000 \\
\hline $\mathrm{H}$ & -4.59940500 & 0.39947500 & -2.40254200 \\
\hline $\mathrm{H}$ & -3.93866500 & -1.60270000 & -0.17731700 \\
\hline
\end{tabular}




\begin{tabular}{|c|c|c|c|}
\hline $\mathrm{H}$ & -5.29062100 & -1.73448200 & -1.29244600 \\
\hline $\mathrm{H}$ & -6.37107500 & 0.16687900 & -0.45874800 \\
\hline $\mathrm{H}$ & -3.62002600 & 0.83876200 & 2.44750000 \\
\hline $\mathrm{H}$ & -3.24639100 & -0.41092000 & 1.35189200 \\
\hline $\mathrm{H}$ & -5.47553200 & 2.16216200 & 2.19461700 \\
\hline $\mathrm{H}$ & -6.72495500 & 1.79644500 & 1.01587000 \\
\hline $\mathrm{H}$ & -2.00093600 & -0.93899800 & -4.73481200 \\
\hline $\mathrm{H}$ & 3.69792500 & 7.85409800 & -1.01977400 \\
\hline $\mathrm{H}$ & 4.90588600 & 6.68495900 & -1.53103100 \\
\hline $\mathrm{H}$ & 3.68430200 & 6.79334300 & 1.27991200 \\
\hline $\mathrm{H}$ & 3.20154300 & 5.67496600 & -0.02714300 \\
\hline $\mathrm{H}$ & 4.65335400 & 3.98434700 & -0.66136500 \\
\hline $\mathrm{H}$ & 6.00151200 & 3.55946800 & 0.35017100 \\
\hline $\mathrm{H}$ & 6.49905800 & 7.59346700 & 0.23563500 \\
\hline $\mathrm{H}$ & -9.29244900 & 4.26812700 & -1.00249600 \\
\hline $\mathrm{H}$ & -10.36707200 & 4.09978000 & -2.39584000 \\
\hline $\mathrm{H}$ & -8.41489100 & 2.76204900 & -2.86797500 \\
\hline $\mathrm{H}$ & -9.67036300 & 1.60894500 & -2.48012300 \\
\hline $\mathrm{H}$ & -11.72662000 & 2.38987400 & -1.11808700 \\
\hline $\mathrm{H}$ & 6.03109900 & -2.70966400 & -1.43488400 \\
\hline $\mathrm{H}$ & 0.19536100 & -2.53452200 & -2.84304200 \\
\hline $\mathrm{H}$ & -1.03548200 & -3.78281900 & -2.59549300 \\
\hline $\mathrm{H}$ & 0.39243000 & -4.13569900 & -3.58700500 \\
\hline $\mathrm{H}$ & -0.31368500 & -1.30532700 & -0.39344600 \\
\hline $\mathrm{H}$ & -0.65789800 & -5.62149800 & -0.88037700 \\
\hline $\mathrm{H}$ & 0.99051300 & -6.04885100 & -0.40902300 \\
\hline $\mathrm{H}$ & 0.46375500 & -6.20839800 & -2.09547400 \\
\hline $\mathrm{H}$ & 2.21880700 & -6.15981100 & -3.09035900 \\
\hline $\mathrm{H}$ & 2.81854000 & -6.85942800 & -1.58769100 \\
\hline $\mathrm{H}$ & 3.91537100 & -6.54623900 & -2.92357900 \\
\hline $\mathrm{H}$ & 5.66520500 & 0.72088900 & -0.38139300 \\
\hline $\mathrm{H}$ & 6.12711100 & -0.95783800 & 0.02301900 \\
\hline $\mathrm{H}$ & 0.31070800 & 0.54239900 & 0.92189600 \\
\hline $\mathrm{H}$ & 3.65297100 & -0.62712000 & 2.35232400 \\
\hline $\mathrm{H}$ & 5.38938500 & 0.97268200 & 3.10367200 \\
\hline $\mathrm{H}$ & 4.95373500 & 3.43262100 & 3.01193500 \\
\hline $\mathrm{H}$ & 2.75541000 & 4.25355500 & 2.23993600 \\
\hline $\mathrm{H}$ & 1.01947400 & 2.66027000 & 1.49135900 \\
\hline $\mathrm{H}$ & 5.28495800 & 8.78396300 & 0.69436400 \\
\hline $\mathrm{H}$ & -11.66439000 & 3.93442500 & -0.24984100 \\
\hline $\mathrm{H}$ & -10.62598900 & 2.58822000 & 0.26004200 \\
\hline $\mathrm{H}$ & -3.32943100 & 0.22389600 & -4.57364400 \\
\hline $\mathrm{H}$ & -1.81805800 & 0.45102600 & -3.65682800 \\
\hline $\mathrm{H}$ & 6.26307300 & -0.36372600 & -1.65594400 \\
\hline
\end{tabular}




$\begin{array}{lrrr}\mathrm{H} & 6.01095300 & 8.84885300 & -0.92246800 \\ \mathrm{C} & 0.28388500 & -3.37677400 & -0.16427900 \\ \mathrm{H} & 0.75303100 & -3.86735900 & 0.69397600 \\ \mathrm{H} & -0.79553600 & -3.51776700 & -0.06415700 \\ \mathrm{C} & 5.82543400 & -5.26990900 & -2.08743100 \\ \mathrm{H} & 5.75493500 & -6.19340800 & -1.50138500 \\ \mathrm{H} & 5.87494400 & -5.56482700 & -3.14371700 \\ \mathrm{H} & 6.78065800 & -4.79783400 & -1.84049300 \\ \mathrm{~N} & 1.43572400 & 2.08452300 & -1.59516300 \\ \mathrm{H} & 0.76197800 & 2.81071400 & -1.79793400 \\ \mathrm{C} & -2.75088900 & -5.99048000 & 4.22899300 \\ \mathrm{C} & -1.91081300 & -4.98909600 & 3.42631100 \\ \mathrm{C} & -2.64236500 & -3.66252500 & 3.17025800 \\ \mathrm{C} & -1.79936100 & -2.63435700 & 2.41547400 \\ \mathrm{O} & -2.27294200 & -1.99607100 & 1.46188300 \\ \mathrm{H} & -0.97522300 & -4.77115500 & 3.95114500 \\ \mathrm{H} & -1.62666300 & -5.43253200 & 2.46295800 \\ \mathrm{H} & -3.56660500 & -3.81790600 & 2.60556600 \\ \mathrm{H} & -2.92073800 & -3.21227400 & 4.13344800 \\ \mathrm{H} & -3.68466200 & -6.23298000 & 3.70868300 \\ \mathrm{H} & -3.01734000 & -5.58229600 & 5.21104400 \\ \mathrm{H} & -2.20596200 & -6.92635600 & 4.39209400 \\ \mathrm{O} & -0.58680900 & -2.50108500 & 2.85703200 \\ \mathrm{H} & 0.06448200 & -1.76783800 & 2.07873300 \\ \mathrm{O} & 2.19833600 & -2.11145400 & 1.72363100 \\ \mathrm{H} & 1.83719000 & -2.83180700 & 2.25721200\end{array}$

\section{Int3b}

$\begin{array}{lrrr}\mathrm{C} & -2.70812100 & -0.18993700 & -3.85966500 \\ \mathrm{C} & -3.45045600 & -1.03592300 & -2.81949300 \\ \mathrm{C} & -4.14170600 & -0.19853400 & -1.73887100 \\ \mathrm{C} & -4.91382000 & -1.05808900 & -0.72554200 \\ \mathrm{~N} & -5.77748300 & -0.24434900 & 0.12332300 \\ \mathrm{C} & -5.46286100 & 0.23068500 & 1.32440500 \\ \mathrm{~N} & -4.34164400 & -0.13252300 & 1.97563000 \\ \mathrm{~N} & -6.33330900 & 1.08171600 & 1.91490800 \\ \mathrm{C} & 5.91482700 & 7.98543800 & -0.32614500 \\ \mathrm{C} & 4.80153200 & 7.06868400 & -0.84409000 \\ \mathrm{C} & 4.02883600 & 6.36306000 & 0.28522400 \\ \mathrm{C} & 4.90841400 & 5.46225500 & 1.15016200 \\ \mathrm{O} & 5.13356300 & 5.70432800 & 2.33500300 \\ \mathrm{~N} & 5.44483100 & 4.39296400 & 0.49318700\end{array}$




\begin{tabular}{|c|c|c|c|}
\hline $\mathrm{C}$ & -11.01672100 & 3.68789100 & -0.42576000 \\
\hline $\mathrm{C}$ & -9.77076200 & 4.09636400 & -1.21977700 \\
\hline $\mathrm{C}$ & -9.00844600 & 2.90721100 & -1.81470300 \\
\hline $\mathrm{C}$ & -8.19401800 & 2.05457900 & -0.81808500 \\
\hline $\mathrm{O}$ & -8.10666200 & 2.45565100 & 0.37775900 \\
\hline $\mathrm{O}$ & -7.65109200 & 1.02269000 & -1.30916300 \\
\hline $\mathrm{C}$ & 4.54300800 & -3.06311000 & -1.42812900 \\
\hline $\mathrm{C}$ & 4.11316000 & -4.32327300 & -1.83393400 \\
\hline $\mathrm{C}$ & 2.75383700 & -4.52848100 & -2.16345000 \\
\hline $\mathrm{C}$ & 3.38087300 & 0.41713300 & -1.29950600 \\
\hline $\mathrm{C}$ & 1.82398300 & -3.47923000 & -2.00893000 \\
\hline $\mathrm{C}$ & 3.26457500 & 2.67309900 & -1.82114600 \\
\hline $\mathrm{C}$ & -0.15015400 & -3.06730900 & -3.54259600 \\
\hline $\mathrm{C}$ & 0.28655700 & -3.58082400 & -2.15165300 \\
\hline $\mathrm{C}$ & 0.09986700 & -1.24940600 & -1.09161900 \\
\hline $\mathrm{O}$ & 3.68522900 & 3.82823700 & -1.78212600 \\
\hline $\mathrm{N}$ & 3.95300000 & 1.59709700 & -1.33159500 \\
\hline $\mathrm{C}$ & 1.42061500 & 1.20291200 & -2.56783700 \\
\hline $\mathrm{O}$ & 0.50965500 & 0.97029100 & -3.33972200 \\
\hline $\mathrm{C}$ & 1.89177000 & 0.24780300 & -1.45587900 \\
\hline $\mathrm{N}$ & 1.43520500 & -1.10033500 & -1.70645400 \\
\hline $\mathrm{C}$ & -0.32253200 & -4.98024600 & -1.91345100 \\
\hline $\mathrm{C}$ & 2.30484800 & -2.18456000 & -1.67258700 \\
\hline $\mathrm{C}$ & 2.37360400 & -5.87591600 & -2.74906000 \\
\hline $\mathrm{C}$ & 3.66125900 & -1.98693300 & -1.36783200 \\
\hline $\mathrm{N}$ & 4.13086900 & -0.67870500 & -1.05384600 \\
\hline $\mathrm{C}$ & 5.53423000 & -0.50056900 & -0.67560100 \\
\hline $\mathrm{C}$ & 0.29282400 & -0.53889300 & 0.27299600 \\
\hline $\mathrm{C}$ & 1.04333100 & 0.74663500 & -0.13663800 \\
\hline $\mathrm{C}$ & 1.82345800 & 1.49241100 & 0.91241700 \\
\hline $\mathrm{C}$ & 2.80497800 & 0.87295100 & 1.70476300 \\
\hline $\mathrm{C}$ & 3.55636200 & 1.62176400 & 2.60949000 \\
\hline $\mathrm{C}$ & 3.34641000 & 2.99570900 & 2.74699400 \\
\hline $\mathrm{C}$ & 2.35994600 & 3.61507100 & 1.97984500 \\
\hline $\mathrm{C}$ & 1.60939400 & 2.86914300 & 1.07163400 \\
\hline $\mathrm{H}$ & -2.74989000 & -1.73184200 & -2.34316700 \\
\hline $\mathrm{H}$ & -4.20353500 & -1.65862400 & -3.32346500 \\
\hline $\mathrm{H}$ & -3.39959200 & 0.40574300 & -1.20051700 \\
\hline $\mathrm{H}$ & -4.84942700 & 0.50045000 & -2.20256300 \\
\hline $\mathrm{H}$ & -4.22704100 & -1.66084900 & -0.12405300 \\
\hline $\mathrm{H}$ & -5.56750700 & -1.75357400 & -1.26590700 \\
\hline $\mathrm{H}$ & -6.57898700 & 0.23606400 & -0.37913300 \\
\hline $\mathrm{H}$ & -4.23490100 & 0.17527100 & 2.92829700 \\
\hline $\mathrm{H}$ & -3.66761800 & -0.86098900 & 1.66041000 \\
\hline
\end{tabular}




\begin{tabular}{|c|c|c|c|}
\hline $\mathrm{H}$ & -5.98240300 & 1.61205600 & 2.69758500 \\
\hline $\mathrm{H}$ & -7.05671600 & 1.57147100 & 1.31895600 \\
\hline $\mathrm{H}$ & -2.24044700 & -0.81979000 & -4.62363700 \\
\hline $\mathrm{H}$ & 4.08945300 & 7.65272500 & -1.44106900 \\
\hline $\mathrm{H}$ & 5.21760800 & 6.31066200 & -1.51671200 \\
\hline $\mathrm{H}$ & 3.57791600 & 7.09779700 & 0.95885700 \\
\hline $\mathrm{H}$ & 3.23420600 & 5.74914400 & -0.15339300 \\
\hline $\mathrm{H}$ & 4.99213600 & 4.06828300 & -0.36089700 \\
\hline $\mathrm{H}$ & 5.84425000 & 3.68365500 & 1.09076600 \\
\hline $\mathrm{H}$ & 6.65275900 & 7.41791700 & 0.25018700 \\
\hline $\mathrm{H}$ & -9.08910600 & 4.64315000 & -0.55925400 \\
\hline $\mathrm{H}$ & -10.06117800 & 4.78226700 & -2.02822300 \\
\hline $\mathrm{H}$ & -8.28858800 & 3.25422000 & -2.56767700 \\
\hline $\mathrm{H}$ & -9.68594700 & 2.23013400 & -2.35092000 \\
\hline $\mathrm{H}$ & -11.73254100 & 3.15101500 & -1.06097600 \\
\hline $\mathrm{H}$ & 5.58978400 & -2.92765500 & -1.18774000 \\
\hline $\mathrm{H}$ & 0.18730400 & -2.04312500 & -3.71871500 \\
\hline $\mathrm{H}$ & -1.24240300 & -3.08530700 & -3.62760800 \\
\hline $\mathrm{H}$ & 0.26119900 & -3.70605200 & -4.33257500 \\
\hline $\mathrm{H}$ & -0.60550600 & -0.68207000 & -1.70723900 \\
\hline $\mathrm{H}$ & -1.40081600 & -4.86445900 & -1.76399200 \\
\hline $\mathrm{H}$ & 0.08523500 & -5.44550000 & -1.01109700 \\
\hline $\mathrm{H}$ & -0.19243000 & -5.66431800 & -2.75194600 \\
\hline $\mathrm{H}$ & 1.64582000 & -5.76887100 & -3.55408800 \\
\hline $\mathrm{H}$ & 1.94925400 & -6.56182000 & -2.00803000 \\
\hline $\mathrm{H}$ & 3.24584200 & -6.36993000 & -3.18008600 \\
\hline $\mathrm{H}$ & 5.66648500 & 0.52595500 & -0.34523000 \\
\hline $\mathrm{H}$ & 5.77088700 & -1.19167400 & 0.13587900 \\
\hline $\mathrm{H}$ & 0.28283700 & 1.42820900 & -0.53029100 \\
\hline $\mathrm{H}$ & 2.95494600 & -0.19390100 & 1.60743800 \\
\hline $\mathrm{H}$ & 4.31526200 & 1.12669700 & 3.21032400 \\
\hline $\mathrm{H}$ & 3.96265700 & 3.59538100 & 3.40905700 \\
\hline $\mathrm{H}$ & 2.19643400 & 4.68395000 & 2.07524700 \\
\hline $\mathrm{H}$ & 0.85613400 & 3.36350100 & 0.46252900 \\
\hline $\mathrm{H}$ & 5.50970500 & 8.76017600 & 0.33496100 \\
\hline $\mathrm{H}$ & -11.53073000 & 4.55856200 & -0.00190700 \\
\hline $\mathrm{H}$ & -10.72433400 & 3.02662500 & 0.39517000 \\
\hline $\mathrm{H}$ & -3.39733900 & 0.49778600 & -4.36383600 \\
\hline $\mathrm{H}$ & -1.91067900 & 0.40441700 & -3.40494900 \\
\hline $\mathrm{H}$ & 6.19732600 & -0.69284500 & -1.52549500 \\
\hline $\mathrm{H}$ & 6.43816600 & 8.48252200 & -1.15035300 \\
\hline $\mathrm{C}$ & -0.30408000 & -2.70718400 & -1.01144400 \\
\hline $\mathrm{H}$ & 0.03221100 & -3.12263200 & -0.05948500 \\
\hline $\mathrm{H}$ & -1.39441100 & -2.76563500 & -1.00377400 \\
\hline
\end{tabular}




$\begin{array}{lrrr}\mathrm{C} & 5.13649500 & -5.43632100 & -1.91731200 \\ \mathrm{H} & 4.81469100 & -6.33470900 & -1.37865100 \\ \mathrm{H} & 5.34217800 & -5.74073900 & -2.95197100 \\ \mathrm{H} & 6.08764200 & -5.11718600 & -1.48105100 \\ \mathrm{~N} & 2.05804600 & 2.42505700 & -2.50854100 \\ \mathrm{H} & 1.69387000 & 3.18076700 & -3.07498300 \\ \mathrm{C} & -3.07230300 & -5.63918200 & 4.55782400 \\ \mathrm{C} & -2.12172700 & -4.67180900 & 3.84222200 \\ \mathrm{C} & -2.81828600 & -3.70909700 & 2.87579400 \\ \mathrm{C} & -1.85665900 & -2.75468800 & 2.13757300 \\ \mathrm{O} & -2.37054000 & -1.89700700 & 1.35322400 \\ \mathrm{H} & -1.55956800 & -4.08416900 & 4.57672600 \\ \mathrm{H} & -1.36368300 & -5.23158300 & 3.28399400 \\ \mathrm{H} & -3.38101400 & -4.26160300 & 2.11068500 \\ \mathrm{H} & -3.56701300 & -3.09638700 & 3.39729000 \\ \mathrm{H} & -3.62042500 & -6.26234600 & 3.84045200 \\ \mathrm{H} & -3.81799200 & -5.09955900 & 5.15480200 \\ \mathrm{H} & -2.52775500 & -6.30921600 & 5.23278800 \\ \mathrm{O} & -0.62618000 & -2.90862100 & 2.35470500 \\ \mathrm{H} & -0.68358100 & -0.29773200 & 0.70961400 \\ \mathrm{O} & 1.06713600 & -1.31926900 & 1.13578500 \\ \mathrm{H} & 0.43608400 & -1.91176900 & 1.64501500\end{array}$

